(C) The British Computer Society 2013. All rights reserved. For Permissions, please email: journals.permissions@oup.com doi:10.1093/comjnl/bxt140

\title{
A Message-Passing Approach to Decentralized Parallel Machine Scheduling
}

\author{
MeritXell Vinyals ${ }^{1, *}$, Kathryn S. Macarthur ${ }^{1}$, Alessandro Farinelli ${ }^{2}$, \\ SARVAPALI D. RAMCHURN ${ }^{1}$ AND NiCHOLAS R. JENNINGS ${ }^{1,3}$ \\ ${ }^{1}$ School of Electronics and Computer Science, University of Southampton, Southampton, UK \\ ${ }^{2}$ University of Verona, Verona, Italy \\ ${ }^{3}$ Department of Computing and Information Technology, King Abdulaziz University, Saudi Arabia \\ ${ }^{*}$ Corresponding author: mv2y11@ecs.soton.ac.uk
}

\begin{abstract}
This paper tackles the problem of parallelizing heterogeneous computational tasks across a number of computational nodes (aka agents) where each agent may not be able to perform all the tasks and may have different computational speeds. An equivalent problem can be found in operations research, and it is known as scheduling tasks on unrelated parallel machines (also known as $R \| C_{\max }$ ). Given this equivalence observation, we present the spanning tree decentralized task distribution algorithm (STDTDA), the first decentralized solution to $R \| C_{\max }$. ST-DTDA achieves decomposition by means of the min-max algorithm, a member of the generalized distributive law family, that performs inference by message-passing along the edges of a graphical model (known as a junction tree). Specifically, ST-DTDA uses min-max to optimally solve an approximation of the original $R \| C_{\text {max }}$ problem that results from eliminating possible agent-task allocations until it is mapped into an acyclic structure. To eliminate those allocations that are least likely to have an impact on the solution quality, ST-DTDA uses a heuristic approach. Moreover, ST-DTDA provides a per-instance approximation ratio that guarantees that the makespan of its solution (optimal in the approximated $R \| C_{\text {max }}$ problem) is not more than a factor $\rho$ times the makespan of the optimal of the original problem. In our empirical evaluation of ST-DTDA, we show that ST-DTDA, with a min-regret heuristic, converges to solutions that are between 78 and $95 \%$ optimal whilst providing approximation ratios lower than 3 .
\end{abstract}

Keywords: parallel machine scheduling; task-allocation; min-max; decentralized coordination; bounded approximation; GDL; junction tree

Received 6 December 2012; revised 3 October 2013

Handling editor: Juan Antonio Rodriguez-Aguilar

\section{INTRODUCTION}

As online and real-time services (e.g. video processing, image classification and route planning) in a range of applications (e.g. social networks, search and rescue and transportation), grow in scale (e.g. hundreds of actors needed to be coordinated during the Haiti earthquake to find victims and hundreds of Mechanical Turk workers needed to execute a project decomposed into micro-tasks), it is crucial that the systems underpinning such services can distribute computation efficiently across the available computational nodes in order to minimize latencies. In the last few years, technologies such as Hadoop, ${ }^{1}$ Amazon's Elastic Compute Cloud $(E C 2)^{2}$ and Ushahidi's CrowdMap platform ${ }^{3}$ have been developed to meet this challenge by distributing computation across, typically heterogeneous, computational nodes such as data centres or mobile devices, possibly belonging to different stakeholders (e.g. government agencies, private companies or individuals), and even human operators (e.g. online crowds on Amazon Turk or volunteers on the ground). Given this heterogeneity

\footnotetext{
${ }^{1}$ http://hadoop.apache.org/.

${ }^{2} \mathrm{http}$ ://aws.amazon.com/ec2/.

${ }^{3}$ http://www.ushahidi.com.
} 
across computational nodes and the variety of tasks that can be requested, it may not be possible (or even desirable) for all nodes to perform all tasks. For example, only nodes equipped with Graphics Processing Units will be able to handle HD video processing tasks, while only Creole-speaking crowd members may be able to do translation tasks in Haiti. Moreover, significant delays may be introduced if hard computational tasks are allocated to the slowest nodes in the system or if the most efficient nodes are overloaded with too many tasks. Therefore, unless such dependencies (i.e. task-specific nodes leading to node eligibility constraints) and limitations (i.e. computational inefficiencies) are factored into the parallel execution of these computational tasks, these distributed information systems may suffer from significant latencies and, in the worst case, failures to deliver real-time services that may cause major financial losses or, in disaster settings, loss of life.

Now, an analogue to this particular class of distributed task allocation problem has been widely studied in Operations Research and is known as scheduling on unrelated parallel machines or $R \| C_{\max }[1,2]$. This problem involves a set of unrelated heterogeneous machines (equivalent to computational nodes), ${ }^{4}$ and a set of computational tasks that must be performed by those computational nodes, potentially under node eligibility constraints (where only a subset of nodes are capable of processing a task). The objective is to find a mapping from tasks to nodes such that the maximum finish time across nodes, known as the makespan, is minimized.

While many algorithms (e.g. [3-5]) have been developed to solve $R \| C_{\max }$, they typically focus on the traditional version of the problem in which a task can be executed by any node. Hence, they do not take account of node eligibility constraints. Moreover, all current approaches require the existence of a central authority that gathers information about all the tasks and all the nodes in the system. This introduces a single point of failure, which is highly undesirable in the context of the online and real-time services exemplified above. In addition, sending all the data to a central authority may be infeasible due to computation or communication constraints. Finally, computational nodes may be managed and owned by different stakeholders that could be unwilling to send such data to a central authority for privacy or security issues.

To overcome these problems, we advocate the design of decentralized algorithms, where both information and computation are distributed across individual nodes in the network, thus avoiding the need of such central authority. In particular, we model the system of distributed computational nodes as a multi-agent system (MAS) (and hence recast the problem as the agent-based $R \| C_{\max }$ problem) where each node is managed by a software agent [6]. Each agent is able to communicate with other nodes to exchange information and

\footnotetext{
${ }^{4}$ Unrelated machines are those in which there is no relation between the execution times of a task on different machines. That is, the time taken by one machine does not affect the time taken by another machine for another task.
}

make decisions about which tasks to run based on its individual computational capacity and load, as well as its capability to run specific types of tasks. Now, since the $R \| C_{\max }$ problem is known to be intractable in the general case [7], we focus on the design of approximate algorithms. Hence, we sacrifice optimality for a practical approach that provides bounds on the error with respect to the optimal solution.

In particular, we develop the spanning tree (ST) decentralized task distribution algorithm (ST-DTDA). ST-DTDA specifies a message-passing protocol for agents to compute high quality approximate solutions in a distributed, efficient manner. By doing so, we advance the state of the art in the following ways:

(i) We provide a novel representation of the (Agent-Based) $R \| C_{\max }$ problem in terms of a junction tree (JT) [8]. This representation is able to capture the constraints on the tasks that each agent can perform, as well as define the communication network used by the agent to exchange messages. Thus, we show that the task allocation constraints result in a sparse network that can be exploited to reduce computation.

(ii) We present the first decentralized task distribution algorithm (i.e. ST-DTDA) for Agent-Based $R \| C_{\max }$ by building upon the min-max algorithm, a standard message-passing algorithm that solves specific optimization problems using the generalized distributive law (GDL) [9]. In particular, ST-DTDA uses a heuristic approach to weight agent-task allocations and thus, eliminates the ones that are least likely to have an impact on the solution quality.

(iii) We show how ST-DTDA can bound the error of its solutions with respect to the optimal solution of the original problem by computing a per-instance approximate ratio. ${ }^{5}$

(iv) We demonstrate the effectiveness of our approach by empirically evaluating ST-DTDA on a range of network structures and using different distributions of task execution times. Our results show that ST-DTDA finds good solutions $(78-95 \%$ of the optimal when using a min-regret heuristic) and outperforms a standard distributed hill-climbing approach in terms of solution quality.

When taken together, our results establish the first benchmarks for distributed solutions to $R \| C_{\max }$ for settings where not all tasks can be performed by all computational nodes.

The rest of this paper is structured as follows. Section 2 gives background on the current approaches used to solve $R \| C_{\max }$ and on the min-max algorithm. Next, Section 3 introduces the Agent-Based $R \| C_{\max }$ problem and maps it to

\footnotetext{
${ }^{5} \mathrm{~A}$ per-instance approximate ratio guarantees that the makespan of its solution is not more than a factor $\rho$ times the makespan of the optimal solution.
} 
a JT representation. Then, in Section 4 we present the STDTDA algorithm. Finally, in Section 5 we present our empirical evaluation and in Section 6 we conclude.

\section{BACKGROUND}

In this section, we provide the foundations of a decentralized solution to the $R \| C_{\max }$ problem. In particular, we first detail the basics of $R \| C_{\max }$ and discuss the relevant literature. Then, we describe the min-max algorithm which we use to develop ST-DTDA.

\subsection{The $R \| C_{\max }$ problem}

This work focuses on finding solutions to a common scheduling problem known as scheduling on unrelated parallel machines $\left(R \| C_{\max }\right)$ [1]. In more detail, $R \| C_{\max }$ requires scheduling a number of tasks on a number of unrelated machines, and assumes that machines are not identical, so that task execution times can differ among them.

Definition $2.1\left(R \| C_{\max }\right)$. Given a set $T$ of tasks, a set $M$ of unrelated machines, and for each $j \in T$ and $i \in M, p_{i j} \in \mathbb{Z}^{+}$, the time taken to process task $j$ on machine $i$, the problem is to schedule the tasks on the machines so as to minimize the makespan. ${ }^{6}$

Now, $R \| C_{\max }$ has been shown to be NP-hard, and hence, exact solution algorithms generally do not scale well [7]. For example, Horowitz and Sahni [3] give an exact algorithm for $R \| C_{\max }$, with a worst-case time complexity exponential in the number of tasks: $\mathcal{O}\left(\min \left\{|T| F,|M|^{|T|}\right\}\right)$ where $F$ is the end time of the schedule obtained by assigning each task to the processor on which its execution time is minimal and $|M|$ is the number of machines. Scheduling each task on the machine with the minimal execution time is unlikely to produce the optimal solution (i.e. the most efficient machines will be overloaded with too many tasks, increasing the global makespan), so the worst-case run-time for this algorithm is exponential.

Thus, in practical applications, it is more beneficial to focus on approximate algorithms to find solutions to $R \| C_{\max }$. For example, Lenstra et al. [5] developed a polynomial-time algorithm with a worst-case performance ratio of 2 (i.e. the solution value is guaranteed to be no more than twice the optimum). Later, Shchepin and Vakhania [4] developed a polynomial-time algorithm for $R \| C_{\max }$ with an improved worstcase bound of $2-1 /|M|$.

Specifically, in this paper, we focus on $R \| C_{\max }$ problems with machine eligibility restrictions (see [10] for a recent survey in the area). This represents a more general and realistic model in which every machine is capable of executing only a subset of the

\footnotetext{
${ }^{6}$ Makespan: the total time needed to complete the whole set of tasks that has been scheduled on one or more machines.
}

tasks (as exemplified in Section 1). Such eligibility constraints can be introduced in a traditional $R \| C_{\max }$ problem by setting the execution time of a task on a given machine to $\infty$ if the machine is cannot perform that task. However, such a direct extension means that existing algorithms cannot exploit the sparsity resulting from eligibility constraints (i.e. each agent can only execute a limited number of tasks). Moreover, despite the existence of good centralized approximation algorithms for $R \| C_{\max }$, there exist no decentralized algorithms as yet.

In contrast, distributed search and constraint handling are a subfield of MASs specifically devoted to this paradigm of decentralized problem solving [11]. Many contributions to this field are message-passing algorithms [12-14], some of which are derived from distributed graphical models representations of the problem (e.g. [13, 14]). To date, however, none of the abovementioned approaches have been applied to/studied for the $R \| C_{\max }$ problem and, as we show in this paper, can potentially provide high quality solutions under certain conditions. Hence, in the next section we describe just such a message-passing algorithm which forms the basis of our approach.

\subsection{The min-max algorithm}

In this section, we introduce the min-max algorithm, as well as the JT structure over which it is executed. Specifically, minmax is a member of the GDL framework, a family of messagepassing algorithms that exploit the way a global function factors into a combination of local functions to compute the objective function in an efficient manner [9]. GDL is defined over two binary operations: marginalization and combination. These define a commutative semiring (see [15] for a more detailed introduction to semirings). In our case, since we are concerned with the problem of minimizing the makespan, such operations are minimization and maximization (the min-max GDL). ${ }^{7}$ In order to guarantee optimality, GDL must operate on a JT [8]. A JT is a popular representation which is used in advanced message-passing algorithms that have been developed in fields such as bioinformatics, image processing and control theory [16]. We define a JT as follows [14].

DEFINITION 2.2. A JT is a tree of cliques that can be represented as a tuple $\langle X, C, S, \Psi\rangle$ where:

(i) $X=\left\{x_{1}, \ldots, x_{n}\right\}$ is a set of variables defined over domains $D_{1}, \ldots, D_{n} ;^{8}$

(ii) $C=\left\{C_{1}, \ldots, C_{m}\right\}$ is a set of cliques such that each clique $C_{i} \subseteq X$

\footnotetext{
${ }^{7}$ Concretely, min-max is based on the commutative semiring $\left\langle\mathbb{R}^{+}, \min , \max , \infty, 0\right\rangle$ where $\mathbb{R}^{+}$is the set of elements of the semiring, $\min$ is the marginalization operator, $\max$ the combination operator, $\infty$ is the neutral element of marginalization ( $\mathrm{min})$ and 0 the neutral element of combination (max).

${ }^{8}$ In general, we will denote $D_{X}$ as the cartesian product of the domain of each individual variable in $X$.
} 
(iii) $S$ is a set of separators, where each separator $S_{i j}$ is an edge between two cliques $C_{i}, C_{j}$ containing the intersection of the variables they represent, i.e. $S_{i j}=$ $C_{i} \cap C_{j} ;$

(iv) $\Psi=\left\{\psi_{1}, \ldots, \psi_{m}\right\}$ is a set of potentials, one per clique, where potential $\psi_{i}$ is a function $\psi_{i}: D_{X_{i}} \rightarrow \mathbb{R}^{+}$whose scope is a subset of the clique to which it is assigned, namely $X_{i} \subseteq C_{i}$.

Furthermore, the running intersection property must hold as follows:

If a variable $x_{i}$ is in two cliques $C_{i}$ and $C_{j}$, then it must also be in all cliques on the (unique) path between $C_{i}$ and $C_{j}$.

We use $\mathbf{x}_{\mathbf{i}}$ throughout to refer to a possible value of variable $x_{i}$, that is, $\mathbf{x}_{\mathbf{i}} \in D_{i}$, and $\mathbf{X}$ to refer to a possible value for each variable in $X$, that is, $\mathbf{X} \in D_{X}$. Moreover, we will refer to $C_{i \backslash j}$ as the set of variables in clique $C_{i}$ excluding those in the clique $C_{j}\left(C_{i \backslash j}=C_{i} \backslash C_{j}=C_{i} \backslash S_{i j}\right)$.

Given a JT, the GDL approach consists of exchanging messages along the edges of the JT. In particular, here we focus on Action-GDL, a specialization of GDL formulated in [14] to efficiently solve distributed constraint optimization problems (e.g. problems in which the objective is to distributedly find the optimal assignment that maximizes the objective function). Action-GDL is executed over a directed $\mathrm{JT}^{9}$ where the cliques are distributed across the set of agents. Here, we assume that each agent $a_{i} \in A$ is assigned a single clique $C_{i}$.

Now, to operationalize min-max using Action-GDL (which we term the min-max algorithm), we propose two messagepassing phases: (1) cost propagation, in which cost messages are sent from leaves up to the root and (2) value propagation, in which optimal assignments are decided and communicated down the tree.

Algorithm 1 outlines the pseudocode for min-max (using Action-GDL). Given a $J T$, each agent $a_{i}$ starts with a tuple $\left\langle C_{i}, S_{i}, \psi_{i}\left(X_{i}\right)\right\rangle$, where $C_{i}$ stands for its clique, $S_{i} \subseteq S$ for the separators relating its clique to adjacent cliques and $\psi_{i}\left(X_{i}\right)$ for its potential function. An agent starts by running the procedure DFS arrangement, a decentralized depth-first search (DFS) algorithm that converts the undirected JT into a directed one by running a token passing mechanism as in [17] (line 1). As the outcome of this DFS, each agent knows its parent $a_{p}$ and its children $\mathrm{Ch}_{i}$ in the directed JT.

After this initialization phase, the agent starts the cost propagation phase (lines 2-7). Each agent $a_{i}$ waits until it receives a cost message from each of its children cliques (line 2). Formally, a cost message $\mu_{j \rightarrow i}$ from agent $a_{j}$ to agent $a_{i}$ is a function that returns a cost for every configuration of variables in their separator $\left(\forall \mathbf{S}_{\mathbf{i j}}\right)$. In the next step, each agent $a_{i}$ computes its local knowledge function, $\kappa_{i}$, by combining (using the max

\footnotetext{
${ }^{9}$ Any undirected JT can be converted into a directed one by picking a root clique and directing arcs from there outwards.
}

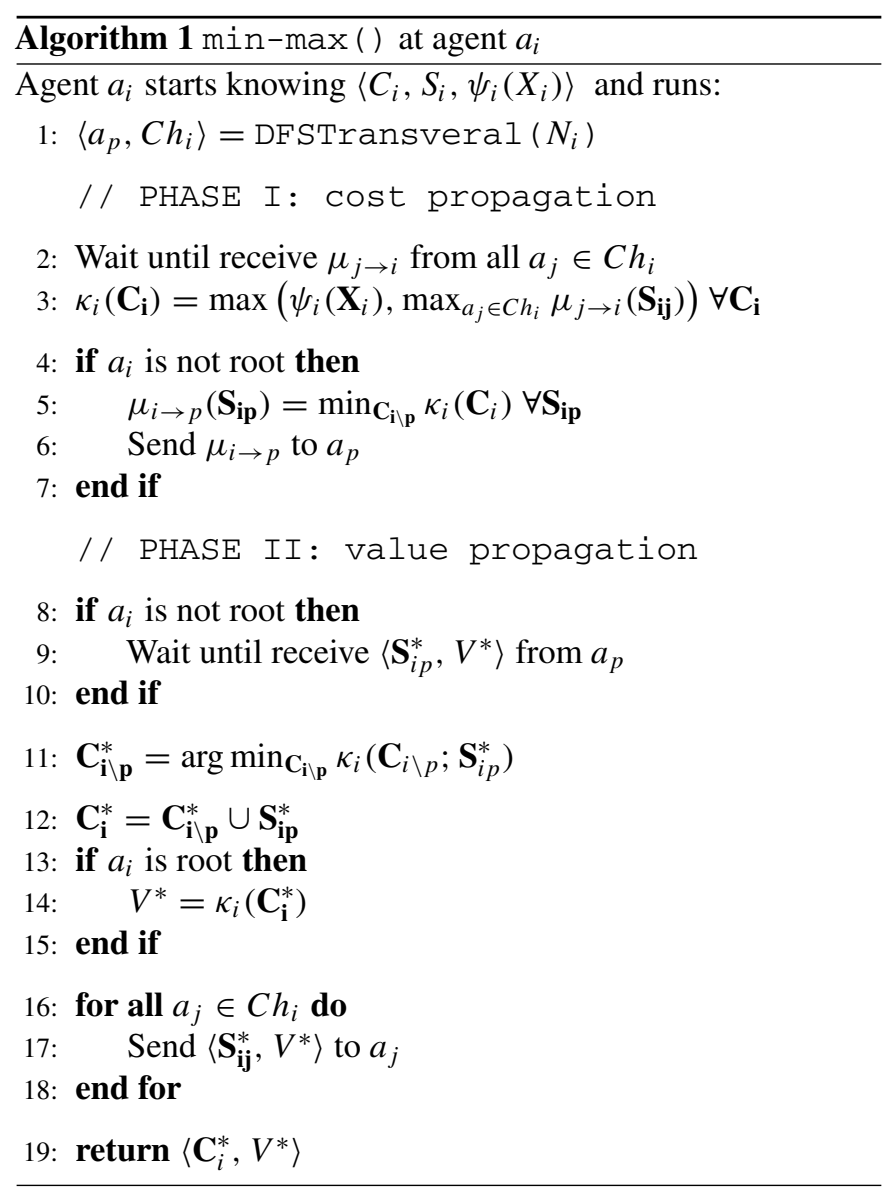

operator) the value of its potential function and all the messages received from its children for every possible assignment of variables in $C_{i}$ (line 3 ). After computing its local knowledge function, if the agent is not the root, it sends a cost message, $\mu_{i \rightarrow p}$, to its parent, $a_{p}$ (lines 4-7). Note that each agent $a_{i}$ computes this message by marginalizing out all variables not common with its parent $a_{p}$ (using the min operator) from its local knowledge function (line 5).

During the value propagation phase (lines 8-18), agents compute the optimal assignment of variables for their clique variables, along with the value of this optimal assignment, and propagate them down the tree. Each agent waits until it receives optimal assignments for all variables it has in common (in the separator) with its parent, $\mathbf{S}_{i p}^{*}$, as well as the value of the optimal assignment, $V^{*}$ (lines $\left.8-10\right)$. Then, the agent fixes the values of these pre-inferred variables in its local knowledge function and computes the assignment that minimizes its local knowledge function for the rest, $\mathbf{C}_{\mathbf{i} \backslash \mathbf{p}}^{*}$ (line 11). At this point, agent $a_{i}$ knows the optimal assignment for all its clique variables (line 12), $\mathbf{C}_{\mathbf{i}}^{*}$. If $a_{i}$ is at the root node, after the cost propagation phase, it can assess the value of the optimal configuration as the value returned by its local knowledge function, $V^{*}$ (lines 13-15). Then, each agent propagates the optimal configuration and its value down the tree to its children 
(lines 16-18). Note, however, that each agent only propagates the variable assignments required by its children's cliques, namely assignments for variables in their respective separators (line 17). Finally, each agent $a_{i}$ returns the values specified in its optimal assignment ${ }^{10}$ for its clique's variables $\left(\mathbf{C}_{\mathbf{i}}^{*}\right)$, being the optimal assignment $\mathbf{X}^{*}$ defined as

$$
\mathbf{X}^{*}=\arg \min _{\forall \mathbf{X}} \max _{a_{i} \in A} \psi_{i}\left(\mathbf{X}_{\mathbf{i}}\right),
$$

where $\mathbf{X}_{\mathbf{i}}$ contains the values assigned by $\mathbf{X}$ to the variables in $X_{i}$.

As stated in [14], we can readily assess the complexity of min-max from cliques' and separators' sizes. In more detail, the local memory/computation required by each agent $a_{i}$ is exponential in the cardinality of its clique (i.e. scales with the joint domain of variables in its clique). However, the number of messages exchanged is linear in the number of edges in the JT and the communication complexity lies in the size of cost messages which are exponential in the cardinality of the respective separators.

Given the basic definitions of $R \| C_{\max }$ and description of the min-max algorithm, we next turn to formulating $R \| C_{\max }$ in a way that we can apply the min-max algorithm.

\section{THE AGENT-BASED $R \| C_{\mathrm{MAX}}$ PROBLEM}

We recast $R \| C_{\max }$ as a multi-agent optimization problem where we have a set of computing entities that we refer to as agents. Hence, from now on, we term the problem the 'Agent-Based $R \| C_{\max }$ ' problem. This general modelling approach allows us to take into account the fact that (i) agents take charge of exchanging messages to find an allocation of computation tasks among them (ii) agents execute these tasks (using the hardware on which they run) and (iii) there may be restrictions on the tasks that each agent can execute. Thus, agents here may represent workers or organizations, as well as machines or processors. Let the set of agents be denoted as $A=\left\{a_{1}, a_{2}, \ldots, a_{|A|}\right\}$, and the set of computational tasks as $T=\left\{t_{1}, t_{2}, \ldots, t_{|T|}\right\}$. Each agent $a_{i}$ can perform a set of tasks $T_{i} \subseteq T$ (recall that a task can be performed by a subset of agents, but not necessarily by all of them). For each agent $a_{i} \in A$, we denote a cost function as $\chi_{i}: T_{i} \rightarrow \mathbb{R}^{+}$, that returns the estimated amount of time it takes for agent $a_{i}$ to perform a task $t \in T_{i}$. Thus, $\chi_{i}\left(t_{k}\right)$ returns the application-specific runtime required for agent $a_{i}$ to perform task $t_{k}$

A graphical representation of an $R \| C_{\text {max }}$ problem in terms of the task dependency network (TDN) is given in Fig. 1. Here, there are four agents (squares) and five tasks (circles). Each agent is connected to the tasks it can potentially perform by edges in the graph, and edges are labelled with $\chi_{i}\left(t_{k}\right)$. Thus, for example, agent $a_{1}$ will incur a runtime of 30 timesteps to

\footnotetext{
${ }^{10}$ In the presence of multiple optimal assignments, the value propagation phase itself ensures that agents converge to the same one.
}

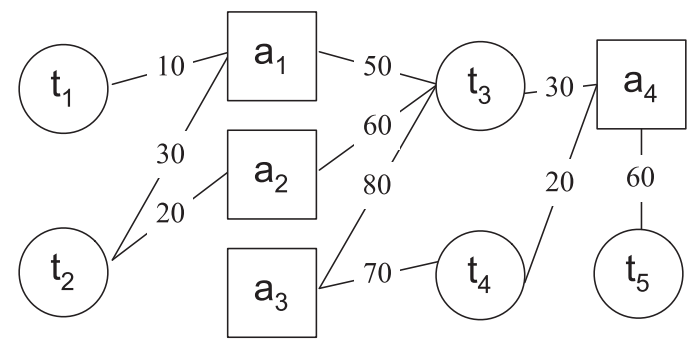

FIGURE 1. Example of a TDN. Agents are represented by squares, tasks by circles and edges between agents and tasks indicate an agent can perform a task (at a cost denoted on the edge).

perform task $t_{2}$ whereas agent $a_{2}$ will only incur a runtime of 20 . Finally, throughout $N_{i}$ will denote the set of agents (neighbours) with which agent $a_{i}$ shares some tasks $\left(N_{i}=\{j \in A, j \neq\right.$ $\left.\left.i \mid \exists t_{k} \in T_{i} \cap T_{j}\right\}\right)$.

Given this, the problem is to schedule all of the tasks in $T$ across the agents in $A$ such that all tasks are completed and the makespan is minimized. Note that this problem is equivalent to $R \| C_{\max }$ (as described in Section 2) but formulated from the decentralized perspective of an MAS. We next formally define the objective of the Agent-Based $R \| C_{\max }$ problem.

\subsection{Objective function}

The objective of Agent-Based $R \| C_{\max }$ is to find a mapping $m: A \rightarrow 2^{T}$ from agents to the set of tasks, such that the makespan is minimized. In particular, we wish to find this mapping subject to a number of constraints. First, each agent may only be assigned tasks that it can execute:

$$
m\left(a_{i}\right) \subseteq T_{i} \quad \forall a_{i} \in A .
$$

Secondly, each task must only be assigned to one agent:

$$
m\left(a_{i}\right) \cap m\left(a_{j}\right)=\emptyset \quad \forall a_{i}, a_{j} \in A, i \neq j .
$$

Thirdly, all tasks must be assigned:

$$
\bigcup_{a_{i} \in A} m\left(a_{i}\right)=T
$$

in which $m\left(a_{i}\right)$ denotes the set of tasks allocated to agent $a_{i}$, under mapping $m$. Given this, our objective is to find a set of optimal mappings $M^{*}$ as follows:

$$
M^{*}=\arg \min _{\forall m} \max _{a_{i} \in A} \sum_{t_{k} \in m\left(a_{i}\right)} \chi_{i}\left(t_{k}\right) .
$$

For instance, Fig. 2 depicts two optimal mappings for the TDN in Fig. 1 where the optimal allocations from agents to tasks are shown with arrows. Thus, the optimal mapping $m^{*}$ in Fig. $2 \mathrm{a}$ is defined as: $m^{*}\left(a_{1}\right)=\left\{t_{1}, t_{2}\right\}, m^{*}\left(a_{2}\right)=\left\{t_{3}\right\}$, $m^{*}\left(a_{3}\right)=\left\{t_{4}\right\}, m^{*}\left(a_{4}\right)=\left\{t_{5}\right\}$ with a makespan value of $\max (10+30,60,70,60)=70$. 
(a)

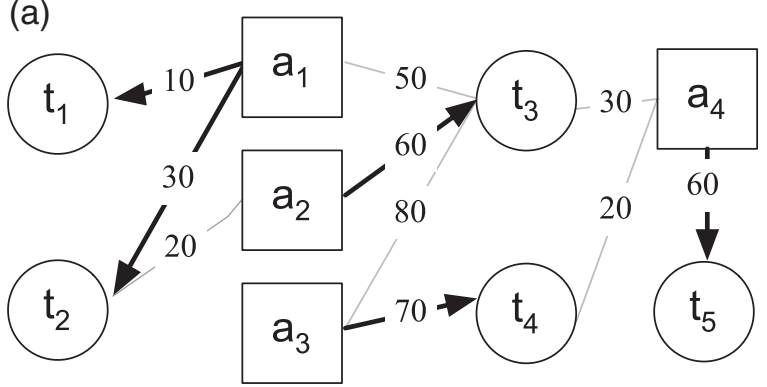

\section{(b)}

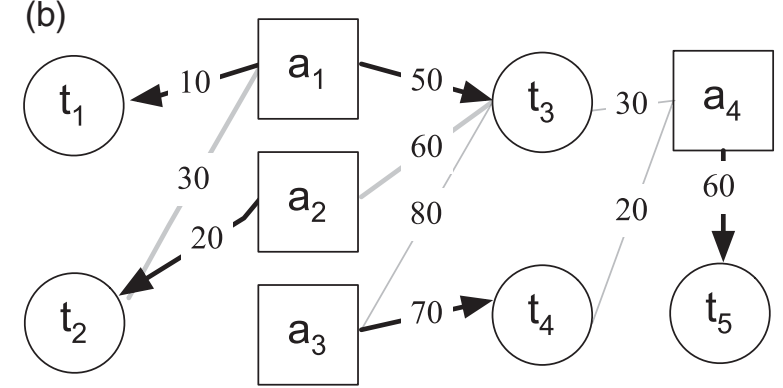

FIGURE 2. Two optimal mappings from agents to tasks with makespan $V^{*}=70$, for the problem in Fig. 1. Arrows between agents and tasks depict an agent being assigned to a task.

Now, in order to solve the objective function given in Equation (2) in a decentralized way, in the next section we show how to represent this problem as a JT over which efficient message-passing algorithms such as the min-max algorithm can operate.

\subsection{JT representation}

To represent an $R \| C_{\max }$ problem as a JT, we need to represent its three components, namely tasks, agents and allocations, in terms of variables, cliques and edges of the JT.

Formally, the JT is built for an $R \| C_{\max }$ problem as follows:

(1) for each task $t_{k} \in T$, a variable $x_{k}$ is created whose domain contains all of the indices of agents that can complete task $t_{k}, D_{k}=\left\{i \mid a_{i} \in A, t_{k} \in T_{i}\right\} ;{ }^{11}$

(2) for each agent $a_{i} \in A$, a clique $C_{i}$ is created containing the variables related to the set of tasks the agent $a_{i}$ can execute, $X_{i}=\left\{x_{k} \mid t_{k} \in T_{i}\right\} ;{ }^{12}$

(3) each clique $C_{i} \in C$ is assigned a potential function encoding the cost function of agent $a_{i}$. Formally,

$$
\psi_{i}\left(X_{i}\right)=\sum_{\substack{t_{k} \in T_{i}, x_{k}=i}} \chi_{i}\left(t_{k}\right)
$$

\footnotetext{
${ }^{11}$ Note that, by doing so, we enforce the constraint that exactly one agent must perform every task.

${ }^{12} x_{k}=i$ means that agent $a_{i}$ is allocated to $t_{k}$.
}

(4) any two cliques $C_{i}$ and $C_{j}$ that share variables are joined by one edge that contains the intersection of their variables, i.e. $S_{i j}=X_{i} \cap X_{j}$.

Now, the graph resulting from applying steps 1-4 above does not always correspond to a JT. This is because the resulting set of edges may not form an acyclic graph (and thus it may form a junction graph, not a JT). Over a junction graph, the minmax algorithm is neither guaranteed to converge nor to find the optimal solution. However, it is always possible to convert this junction graph to a JT, at the expense of increasing the size and number of cliques and separators. Thus, to convert the junction graph to a JT we can apply the following steps $[18,19]:^{13}$

(5) select a subset of edges that form a connected tree among cliques;

(6) enlarge cliques (and the corresponding separators) until the running intersection property is satisfied (by running a distributed protocol such as the one described in [18]).

In what follows, we provide an example of how the steps above are applied to a TDN.

Fig. 3a depicts the graph resulting from applying steps 14 to the TDN from Fig. 1. In more detail, the graph contains five variables, $\left\{x_{1}, x_{2}, x_{3}, x_{4}, x_{5}\right\}$ that correspond to the five tasks in the figure. Each agent $a_{i}$ 's clique, denoted by a large circle, contains all variables in $X_{i}$. Thus, the set of variables corresponding to agent $a_{2}$ 's clique, $X_{2}$, is composed of $x_{2}$ and $x_{3}$, which are the two tasks that $a_{2}$ can perform in Fig. 1. The domain of $x_{2}$ is composed of two values, namely 1 and 2 , corresponding to the indices of the agents that can perform task $2, a_{1}$ and $a_{2}$. Moreover, Fig. 3 depicts the potential function of agent $a_{4}, \psi_{4}$, in a tabular form (listing one entry per configuration of variables). Thus, $\psi_{4}$, defined over variables $x_{3}, x_{4}$ and $x_{5}$, returns a runtime of 80 for the configuration $x_{3}=3, x_{4}=4$ and $x_{5}=4$, which is the runtime incurred by $a_{4}$ to complete tasks 4 and 5 in Fig. 1 . Edges are labelled with the intersection of two cliques. Thus, agent $a_{2}$ is linked to $a_{3}$ by an edge that contains the only common variable in their cliques: $x_{3}$. As we can see, this junction graph is not acyclic and thus, steps 5 and 6 are needed.

Fig. $3 \mathrm{~b}$ shows the JT resulting from applying steps 5 and 6 over the junction graph in Fig. 1. We observe that from the set of edges in the initial junction graph, only those joining the clique of agent $a_{4}$ with the rest of cliques have been selected to compose the JT. Moreover, the clique of agent $a_{4}$ has been enlarged to include variable $x_{2}$ in order to satisfy the running intersection property (e.g. $x_{2}$ is already included in the cliques of $a_{1}$ and $a_{2}$ and so it should be also included in the clique of $a_{3}$ because the latter is in the path connecting the first two).

Now, this representation allows the application of the minmax algorithm to find an optimal solution to Agent-Based $R \| C_{\max }$ in a decentralized fashion. However, the complexity of

\footnotetext{
${ }^{13}$ Note that if the TDN is acyclic, then steps 5 and 6 are never needed since the junction graph that results from applying steps 1-4 is guaranteed to be a JT.
} 


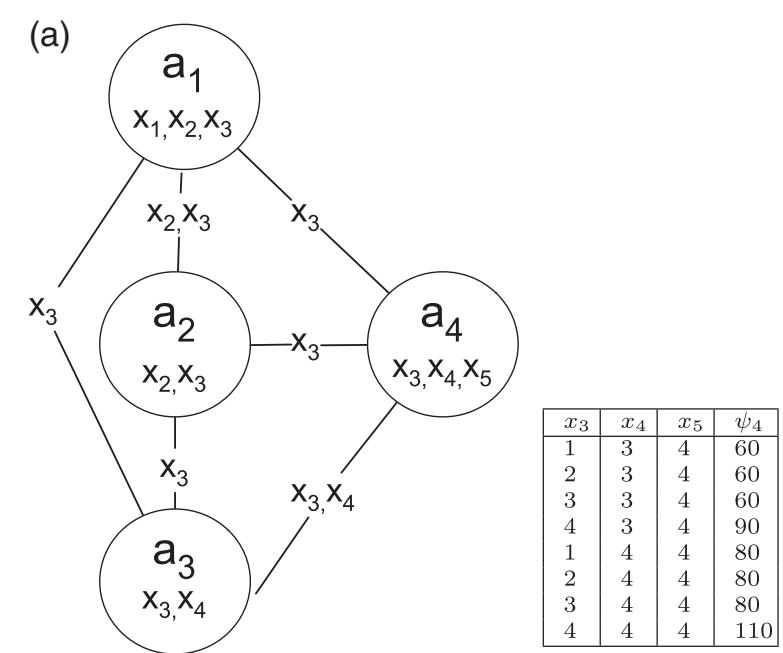

Junction graph generated after steps 1-4.

(b)

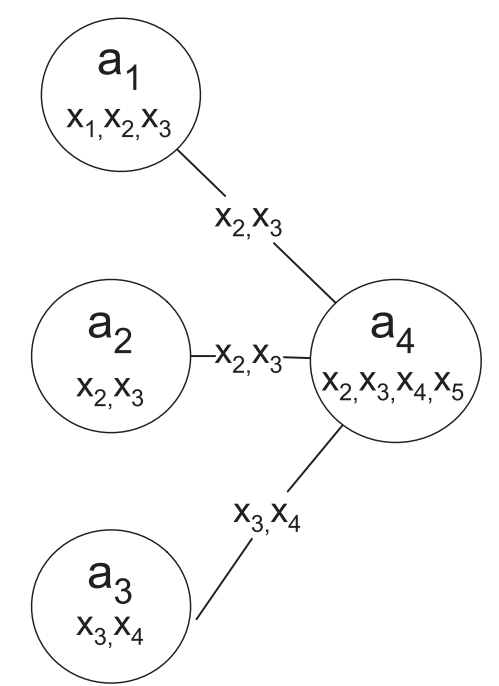

Junction tree generated after steps 5-6 are applied on (a).

FIGURE 3. A JT representation of the scenario given in Fig. 1. Large circles are cliques, with the elements of the cliques listed. Edges are labelled with common variables between cliques.

running min-max over this JT is exponential in the cardinality of the largest clique (see Section 2.2). This renders this approach computationally intractable in the general case where all agents can perform an unrestricted number of tasks. Crucially, in the particular case when the TDN is acyclic, the computational complexity grows exponentially in the number of tasks which $a_{i}$ is connected to in the TDN. Since, by definition, the cardinality of an agent's clique is bounded below by the number of tasks that an agent is connected to $\left(\left|C_{i}\right| \geq T_{i}\right)$, the computational complexity of the algorithm will also be bounded by this. Hence, the main contribution of this paper, which we detail in the next section, is the formulation of solutions that exploit this sparsity in acyclic TDNs that have bounded agent degrees.

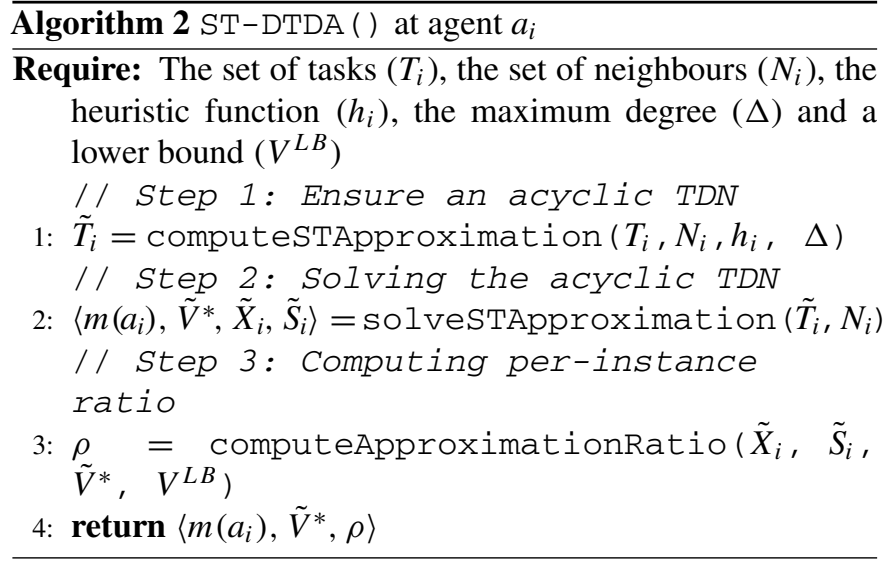

\section{THE ST-DTDA ALGORITHM}

In this section, we describe ST-DTDA, an approximate algorithm to solve the Agent-Based $R \| C_{\max }$ problem. The key feature of ST-DTDA involves a step that prunes the JT representation of the problem in order to allow the minmax algorithm to converge to high quality solutions with low computational cost. ${ }^{14}$ Moreover, this allows to provide a bound on the quality of the solution computed. In more detail, STDTDA prunes some agent-task allocations so as to form an acyclic TDN where the maximal number of tasks connected to an agent is bounded by a parameter $\Delta .{ }^{15}$ It then applies the min-max algorithm to compute a high quality solution as well as a worst case bound on it.

Specifically, ST-DTDA consists of three steps (outlined in Algorithm 2):

(1) Ensure an acyclic TDN-ST-DTDA agents create an acyclic TDN by finding an ST of the original problem with a bounded number of tasks per agent and omitting all agent-task allocations not contained in the ST.

(2) Solving the acyclic TDN-ST-DTDA agents run minmax to optimally solve the JT that encodes the acyclic TDN created in step 1 and find an approximate solution for the $R \| C_{\max }$ problem.

(3) Computing the per-instance approximation ratio-STDTDA agents execute a third message-passing phase to compute a worst-case bound $(\epsilon)$ on the distance between the quality of the optimal solution in the original problem and the quality of the solution of the ST-approximate problem. Then, ST-DTDA agents use this error bound and a lower bound on the optimal

\footnotetext{
${ }^{14}$ This approach was demonstrated for other algorithms from the GDL family [20], adding a preprocessing step that transforms the original problem to one that is acyclic, can reduce the computational complexity.

${ }^{15}$ Only the tasks that can potentially be executed by at least two agents are considered when computing the agent's degree. Single-connected tasks can be omitted from the TDN by adding their execution times to the initial agent's makespan.
} 
solution value to compute a per-instance approximation ratio.

After running ST-DTDA, each agent knows the set of tasks allocated to it, $m\left(a_{i}\right)$, as well as the makespan, $\tilde{V}^{*}$, and a perinstance approximation ratio, $\rho$, of its allocation. We elaborate further on these steps in the remainder of this section.

\subsection{Ensuring an acyclic TDN}

In this step, the agents build an ST of the TDN with a bounded number of tasks per agent. The key issue is that producing any ST of the TDN involves ignoring some of the potential agenttask allocations (i.e. it produces an acyclic approximation of the original network). Therefore, it is crucial that the possible solutions in the ST are of high quality_preferably containing the optimal solution for the original network and, if not, a solution whose makespan is close to that of the optimal. With this aim, agents assign a weight to each agent-task allocation in the TDN that estimates its impact on the solution makespan. Next, agents eliminate the agent-task allocations that are less likely to generate low makespans. However, since computing the exact values for these weights is as difficult as solving the original problem, ST-DTDA uses a heuristic approach to approximate them. Therefore, in this step, agents assign a weight $\omega_{i k} \in \mathbb{R}^{+}$to each of the edges that have not already been included in the ST.

First, we detail the particular distributed procedure that STDTDA agents follow to compute the ST approximation of the TDN in which each agent is connected to at most $\Delta$ tasks. ${ }^{16}$ Secondly, we propose three different heuristics functions to weight the edges of the TDN.

\subsubsection{Decentralized ST approximation computation}

Using Algorithm 3, ST-DTDA agents build the ST iteratively, adding, at each iteration, the edge with minimal weight to the ST by implementing a distributed version of Kruskal's Maximum ST algorithm [21]. ${ }^{17}$ In more detail, Algorithm 3 outlines the computeSTApproximation procedure. Each agent is given the set of tasks it can potentially execute $\left(T_{i}\right)$, the set of neighbours with which $a_{i}$ shares some tasks $\left(N_{i}\right)$, a heuristic edge weighting function $\left(h_{i}\right)$ and the maximum number of tasks per agent $(\Delta)$. An agent starts by running the procedure DFSTransveral to compute a directed agentinduced ST that agents will use as a (directed) communication tree (line 1). As the outcome of this DFS, each agent knows its parent $a_{p}$ and its children $\mathrm{Ch}_{i}$ in the tree. Then, the agent initializes the set of tasks to which it is connected in the ST,

\footnotetext{
${ }^{16}$ This is excluding single-connected tasks: $\Delta$ does not count singleconnected tasks.

${ }^{17}$ The computeSTApproximation procedure can be seen as a distributed version of Kruskal's algorithm with the difference that in Kruskal's algorithm all weights are assumed static during the ST computation whereas here the weights change depending on the set of edges already added to the ST.
}

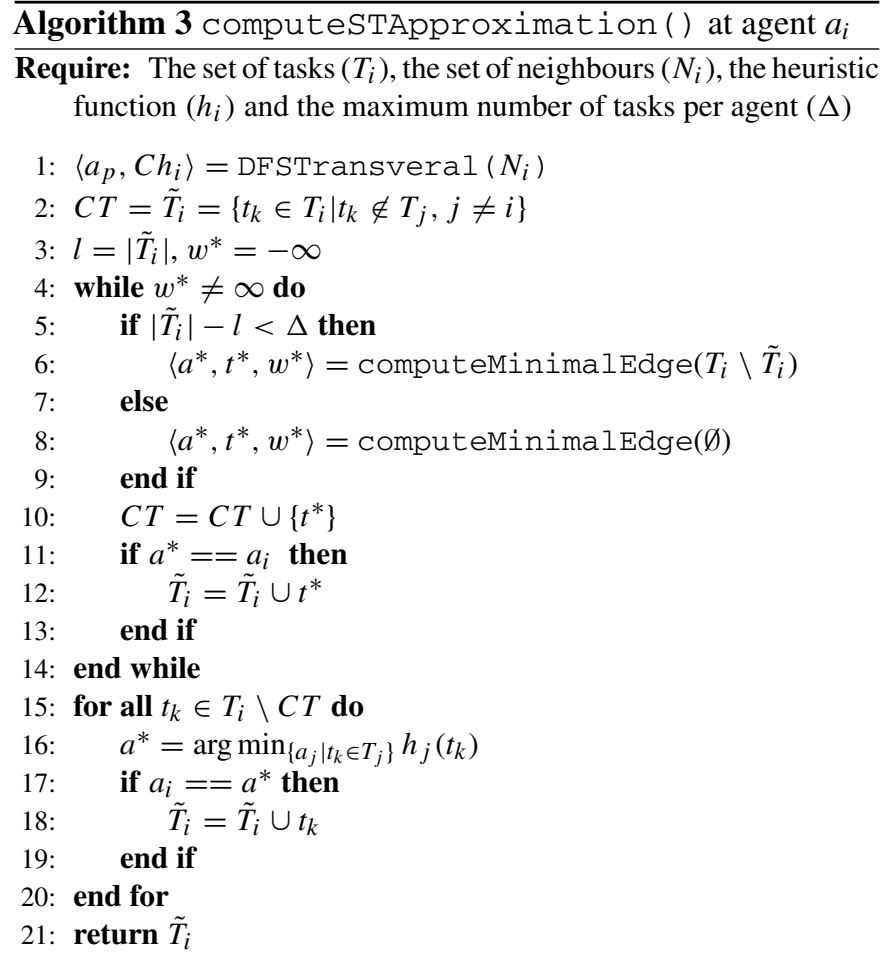

denoted as $\tilde{T}_{i}$, and the set of connected tasks, denoted as CT, as the set of tasks for which $a_{i}$ is the only agent that can execute them (line 2). Although the edges between $a_{i}$ and these tasks will be included in the ST-TDN anyway, adding them initially helps $a_{i}$ to predict its final makespan with the heuristic. Moreover, each agent keeps track of this number of initial single-connected tasks because they are not considered when bounding the agent's degree by $\Delta$ (line 3$)$.

Then, each agent iterates over the main loop of this step until no further edge can be added to the ST (lines 4-14), finding at each iteration the not added edge with minimal weight by repetitive calls to computeMinimalEdge (line 19). This procedure takes as an input the set of tasks whose edges with the agent can be considered as minimal. If the number of tasks that it is connected to does not exceed the maximum (line 5), then this set of tasks correspond to the tasks not added up to that iteration (line 6). Otherwise, the agent calls computeMinimalEdge with the empty set to avoid being linked to more tasks (line 7). Finally, the agent $a_{i}$ adds to its set of tasks all tasks that have not been connected so far and for which $a_{i}$ is the agent with the lowest task execution time (lines 15-20). Note that the inclusion of these tasks does not increase $\Delta$ since agent $a_{i}$ will be the only agent connected to them in the ST. At the end of this phase, each agent returns the set of tasks to which it is connected in the ST, $\tilde{T}_{i}$.

Up to this point, we have described this ST-DTDA step independently of the heuristic edge weighting function used. Hence, for the sake of completeness, in the next section we 
propose a number of heuristic edge weighting functions and illustrate their operation using as a running example a trace of ST-DTDA over the TDN depicted in Fig. 1.

\subsubsection{Heuristic edge weighting functions}

We propose three different heuristics to weight edges of the TDN, namely the best-case, the worst-case and the min-regret heuristics. Each heuristic explores an alternative method for encoding the impact of edges on the final makespan. The bestcase heuristic is a myopic heuristic that simply weights each agent-task allocation with the corresponding task processing time. As a result, we use it as a baseline for our approach. The worst-case and the min-regret heuristics incorporate information regarding the expected agent workload in their weights. Moreover, min-regret aims to improve over worstcase by bringing forward the decision regarding the agent-task allocations with higher impact by minimizing the regret (i.e. the potential to increase the makespan should the wrong edges be pruned) instead of the processing time. In what follows, all three weighting heuristics are formulated from the point of view of the agents. Thus, heuristic $h_{i}$ is a function that given a task $t_{k} \in T_{i}$ returns the weight for the edge between $a_{i}$ and $t_{k}$, namely $w_{i k}$.

The best-case heuristic. This simply weights the edge between $a_{i}$ and $t_{k}$ with the time it takes agent $a_{i}$ to compute task $t_{k}$. Formally,

$$
h_{i}\left(t_{k}\right)=\chi_{i}\left(t_{k}\right)
$$

Note that the weights given by this heuristic are independent from the edges already added to the ST. The intuition behind this is that by removing high-weight edges we would remove the most costly agent-task allocations, which are the least likely to be in the optimal solution. We refer to this heuristic as best-case since it assumes that the only task that will be allocated to $a_{i}$ is $t_{k}$. Thus, the ST that results from this heuristic always encodes the greedy allocation, $\check{m}$, that assigns each task to the agent with the lowest task execution time (i.e. the edge from one task to the agent with minimal execution time is always included).
In general, an ST resulting from this heuristic is likely to encode any solution that assigns tasks to one of the first $n$th agents in the order of its processing time, where $n$ is a small number.

As an example, Fig. 4a shows a trace of the weights computed at each iteration of the computeSTApproximation procedure and Fig. $4 \mathrm{~b}$ the edges included in the ST at the end of this procedure when using the best-case heuristic. Since the value returned by this heuristic does not depend on the set of edges already included in the ST, the weight for an edge does not change between iterations. At the initialization step (iteration 0 ), edges $\left(a_{1}, t_{1}\right)$ and $\left(a_{4}, t_{5}\right)$ are added to the ST, as a result of preprocessing tasks with a single neighbour. During the first iteration, the edge between $a_{2}$ and $t_{2}$ has a starting minimal weight (20) and it is added to the ST. The second edge to be added is $a_{4}$ to $t_{4}$, then $a_{4}$ to $t_{3}, a_{1}$ to $t_{2}, a_{1}$ to $t_{3}$, and finally $a_{3}$ to $t_{4}$. Observe that all edges from one task to the agent with minimal execution time, namely $\left(t_{1}, a_{1}\right),\left(t_{2}, a_{2}\right),\left(t_{3}, a_{4}\right)$ and $\left(t_{4}, a_{4}\right)$, have been added. In the same way, all the edges form one task to the agent with second minimal execution time have also been added (i.e. $\left(t_{2}, a_{1}\right),\left(t_{3}, a_{1}\right)$ and $\left.\left(t_{4}, a_{3}\right)\right)$. Thus, for this particular example, the ST encodes any solution that assigns tasks either to the first or the second agent with minimal execution time for that task and hence, it encodes one of the optimal solutions. Thus, for example, the set $\tilde{T}_{2}$ (related to agent $a_{2}$ ) is composed of $t_{2}$ (for which $a_{2}$ is the agent with second minimal execution time) but not of $t_{3}$ (for which it is the third) since this edge is pruned in the ST.

The worst-case heuristic. The worst-case heuristic aims to improve upon the greedy-like behaviour of the best-case to consider the worst possible allocation an agent could get. Thus, at each iteration, this heuristic considers not only the processing time of a task by an agent but also the time taken for tasks that will be allocated to it in the current iteration.

Formally, let $m^{s}$ be the best allocation for tasks added up to iteration $s$, namely the optimal allocation of tasks already included up to iteration $s . m^{s}$ is computed at each iteration by running min-max over the acyclic TDN that form the allocations included up to iteration $s$. Then, the worst-case heuristics (a)

\begin{tabular}{cccccccc} 
& 0 & 1 & 2 & 3 & 4 & 5 & 6 \\
\hline$\left(a_{1}, t_{1}\right)$ & $\mathbf{1 0}$ & - & - & - & - & - & - \\
$\left(a_{1}, t_{2}\right)$ & 30 & 30 & 30 & - & $\mathbf{3 0}$ & - & - \\
$\left(a_{1}, t_{3}\right)$ & 50 & 50 & 50 & 50 & 50 & $\mathbf{5 0}$ & - \\
$\left(a_{2}, t_{2}\right)$ & 20 & $\mathbf{2 0}$ & - & - & - & - & - \\
$\left(a_{2}, t_{3}\right)$ & 60 & 60 & 60 & 60 & 60 & 60 & - \\
$\left(a_{3}, t_{3}\right)$ & 80 & 80 & 80 & 80 & 80 & 80 & 80 \\
$\left(a_{3}, t_{4}\right)$ & 70 & 70 & 70 & 70 & 70 & 70 & $\mathbf{7 0}$ \\
$\left(a_{4}, t_{3}\right)$ & 30 & 30 & 30 & $\mathbf{3 0}$ & - & - & - \\
$\left(a_{4}, t_{4}\right)$ & 20 & 20 & $\mathbf{2 0}$ & - & - & - & - \\
$\left(a_{4}, t_{5}\right)$ & $\mathbf{6 0}$ & - & - & - & - & - & - \\
\hline
\end{tabular}

Weights computed for not added edges at each iteration. The weight of the edge added in a given iteration appears boldfaced. (b)

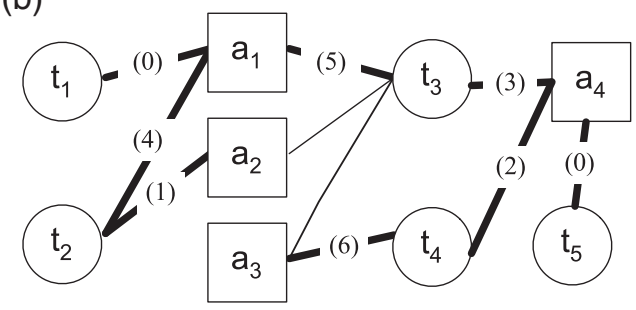

ST-TDN. Boldfaced edges are those included in the ST, each labeled with the number of the iteration at which it has been included.

FIGURE 4. Example of (a) the weights of not added edges and (b) the acyclic TDN computed by ST-DTDA using the best-case heuristic over the TDN depicted in Fig. 1. 
(a)

\begin{tabular}{cccccccc} 
& 0 & 1 & 2 & 3 & 4 & 5 & 6 \\
\hline$\left(a_{1}, t_{1}\right)$ & $\mathbf{1 0}$ & - & - & - & - & - & - \\
$\left(a_{1}, t_{2}\right)$ & 40 & 40 & $\mathbf{4 0}$ & - & - & - & - \\
$\left(a_{1}, t_{3}\right)$ & 60 & 60 & 60 & $\mathbf{6 0}$ & - & - & - \\
$\left(a_{2}, t_{2}\right)$ & 20 & $\mathbf{2 0}$ & - & - & - & - & - \\
$\left(a_{2}, t_{3}\right)$ & 60 & 60 & 80 & 80 & - & - & - \\
$\left(a_{3}, t_{3}\right)$ & 80 & 80 & 80 & 80 & 80 & 150 & 150 \\
$\left(a_{3}, t_{4}\right)$ & 70 & 70 & 70 & 70 & $\mathbf{7 0}$ & - & - \\
$\left(a_{4}, t_{3}\right)$ & 90 & 90 & 90 & 90 & 90 & 90 & $\mathbf{9 0}$ \\
$\left(a_{4}, t_{4}\right)$ & 80 & 80 & 80 & 80 & 80 & $\mathbf{8 0}$ & - \\
$\left(a_{4}, t_{5}\right)$ & $\mathbf{6 0}$ & - & - & - & - & - & - \\
\hline
\end{tabular}

Weights computed for not added edges at each iteration. The weight of the edge added in a given iteration appears boldfaced. (b)

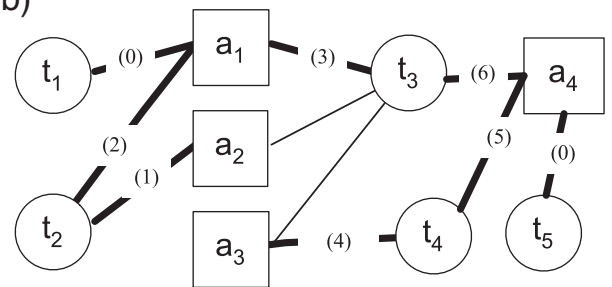

ST-TDN. Boldfaced edges are those included in the ST, each labeled with the number of the iteration at which it has been included.

FIGURE 5. Example of (a) the weights of not added edges and (b) the acyclic TDN computed by ST-DTDA using the worst-case heuristic over the TDN depicted in Fig. 1.

weights the edge between $a_{i}$ and $t_{k}$ at an iteration $s$ with the time required by $a_{i}$ to execute task $t_{k}$ plus the total time it would take agent $a_{i}$ to compute all tasks allocated to it in the current optimal allocation, $m^{s}\left(a_{i}\right)$. Formally,

$$
h_{i}\left(t_{k}\right)=\chi_{i}\left(t_{k}\right)+\sum_{\substack{t_{l} \in m^{s}\left(a_{i}\right) \\ l \neq k}} \chi_{i}\left(t_{l}\right) .
$$

In case of ties, we consider the edge with the highest execution time to be the heaviest. We refer to this heuristic as worstcase because the weight $w_{i k}$ it computes corresponds to the runtime incurred at agent $a_{i}$ when allocated $t_{k}$ and all other tasks assigned in the current ST.

As an example, Fig. 5a shows a trace of the weights computed at each iteration of the computeSTApproximation procedure and Fig. 5b the edges included in the ST at the end of this procedure when using the worst-case heuristic. At the initialization step, all weights are computed as in the best-case heuristic with the exception of those connected to agent $a_{1}$ and $a_{4}$ that need to add the cost of edges already included as a result of preprocessing tasks with a single neighbour. Then, the first edge to be added is $\left(a_{2}, t_{2}\right)$ because it is the one with starting minimal weight (20) and the weights not added edges will be updated (the weight of the edge between $a_{2}$ to $t_{3}$ is updated to 80 for the next iteration). The next edge to be added is $a_{1}$ to $t_{3}$, then $a_{3}$ to $t_{4}, a_{4}$ to $t_{4}$ and finally $a_{4}$ to $t_{3}$. Note that, for this particular example, worst-case adds the same edges than bestcase but in different priority order. Hence, the edge between $a_{4}$ and $t_{4}$ is added at the fifth iteration instead of the second since this edge is much less attractive when you consider that agent $a_{4}$ is already assigned $t_{5}$.

The min-regret heuristic. An obvious problem with the worstcase heuristic is that it often postpones the decision regarding the most crucial agent-task allocations to the last iterations where there is no much freedom of action. The min-regret tries to circumvent this problem by prioritizing allocations based on the cost difference of allocating a task to its best agent or to its second best agent. In other words, min-regret aims to minimize the regret when omitting edges. Here regret is defined as the difference in terms of cost that thee system would incur if $t_{k}$ is not allocated to $a_{i}$, but rather allocated to another agent other than $a_{i}$. The cost of allocating a task $t_{k}$ to an agent $a_{i}$ is computed as in the worst-case heuristic: the agent will process task $t_{k}$ and all other tasks to which it is assigned in the best current allocation in the ST. Formally,

$$
\begin{aligned}
h_{i}\left(t_{k}\right)= & \chi_{i}\left(t_{k}\right)+\sum_{\substack{t_{l} \in m^{s}\left(a_{i}\right) \\
l \neq k}} \chi_{i}\left(t_{l}\right) \\
& -\min _{\substack{j \in D_{k} \\
j \neq i}}\left(\chi_{j}\left(t_{k}\right)+\sum_{\substack{t_{l} \in m^{s}\left(a_{j}\right) \\
l \neq k}} \chi_{j}\left(t_{l}\right)\right),
\end{aligned}
$$

where $m^{s}$ is the best allocation for tasks added up to iteration $s$.

In cases where two edge weights are equal, we break ties in the same way as we do for the worst-case heuristic.

As an example, Fig. 6 shows the resulting ST-TDN and a trace of the weights computed for possible not added edges at each iteration of the computeSTApproximation procedure when using the min-regret heuristic. At the initialization step (iteration 0 ), all weights are computed and edges $\left(a_{1}, t_{1}\right)$ and $\left(a_{4}, t_{5}\right)$ are included as a result of preprocessing tasks with a single neighbour. Thus, in the first step, the weight between agent $a_{2}$ and task $t_{2}$ is -20 since 30 is the execution time of task $t_{2}$ at $a_{2}$ and the alternative best allocation is $t_{2}$ to $a_{1}$ which has a cost of 30. At this step, this weight is the lowest and hence the edge is included in the ST. In the following steps, the weights are recomputed being the next edge to be added $a_{1}$ to $t_{3}$, then $a_{3}$ to $t_{4}$, then $a_{4}$ to $t_{4}$, then $a_{2}$ to $t_{3}$ and finally $a_{4}$ to $t_{3}$. Note that unlike the best-case and worst-case heuristic, min-regret does not prioritizes the inclusion of the edge between $a_{1}$ and $t_{2}$ which is not finally added to the ST. This is because the edges regarding task $t_{3}$ have higher regret and $t_{4}$ have higher regret than not the edge between $a_{1}$ and $t_{2}$ (there is higher difference on the solution impact between their possible allocations).

Now, independently of the heuristic function used, once we have found the ST, we can describe the acyclic TDN as follows: for each agent $a_{i}$, we have $\tilde{T}_{i}$ as the set of tasks to which agent $a_{i}$ is connected in the ST. Given this, it is now possible to find 
(a)

\begin{tabular}{lccccccc} 
a) & 0 & 1 & 2 & 3 & 4 & 5 & 6 \\
\hline$\left(a_{1}, t_{1}\right)$ & $\infty$ & - & - & - & - & - & - \\
$\left(a_{1}, t_{2}\right)$ & 20 & 20 & 20 & 70 & 70 & 70 & - \\
$\left(a_{1}, t_{3}\right)$ & 0 & 0 & $\mathbf{- 2 0}$ & - & - & - & - \\
$\left(a_{2}, t_{2}\right)$ & -20 & $\mathbf{- 2 0}$ & - & - & - & - & - \\
$\left(a_{2}, t_{3}\right)$ & 0 & 0 & 20 & 20 & 20 & $\mathbf{2 0}$ & - \\
$\left(a_{3}, t_{3}\right)$ & 20 & 20 & 20 & 20 & 90 & 90 & 90 \\
$\left(a_{3}, t_{4}\right)$ & -10 & -10 & -10 & $\mathbf{- 1 0}$ & - & - & - \\
$\left(a_{4}, t_{3}\right)$ & 30 & 30 & 30 & 30 & 30 & 30 & $\mathbf{3 0}$ \\
$\left(a_{4}, t_{4}\right)$ & 10 & 10 & 10 & 10 & $\mathbf{1 0}$ & - & - \\
$\left(a_{4}, t_{5}\right)$ & $-\infty$ & - & - & - & - & - & - \\
\hline
\end{tabular}

Weights computed for not added edges at each iteration. The weight of the edge added in a given iteration appears boldfaced. (b)

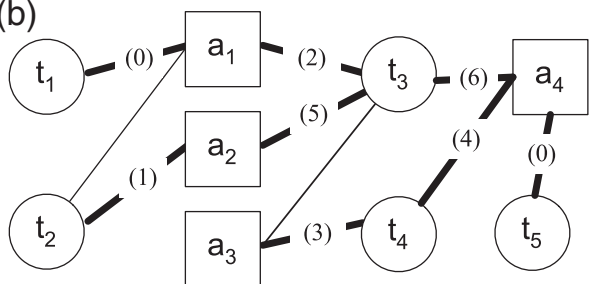

ST-TDN. Boldfaced edges are those included in the

ST, each labeled with the number of the iteration at which it has been included.

FIGURE 6. Example of (a) the weights of not added edges and (b) the acyclic TDN computed by ST-DTDA using the min-regret heuristic over the TDN depicted in Fig. 1.

a mapping $m^{*}$ that minimizes the makespan incurred by tasks in $\tilde{T}_{i}$ for any agent $a_{i}$.

\subsection{Solving the acyclic TDN with min-max}

In this step, agents run min-max on the JT representation of the acyclic TDN computed in step 1. As discussed in Section 3.2, any acyclic TDN maps directly to a distributed JT and hence ST-DTDA agents only need to execute steps $1-4$ to encode the acyclic TDN into a JT.

In more detail, Algorithm 4 outlines the solveSTApproximation procedure that each ST-DTDA agent executes during this step. As input, each agent receives the set of tasks that it is connected to in the ST $\left(\tilde{T}_{i}\right)$ and the set of neighbours that $a_{i}$ shares some tasks $\left(N_{i}\right)$ with. Each agent starts by exchanging this set of tasks with its neighbours (lines $1-4)$. Once an agent has received the set of tasks from all its neighbours, it proceeds to create the corresponding JT. First, an agent creates one variable $x_{k}$ for task $t_{k}$ in $\tilde{T}_{i}$ whose domain contains the set of indexes of all agents that can execute $t_{k}$ in the ST (lines 5-7). Secondly, the agent sets its clique and its

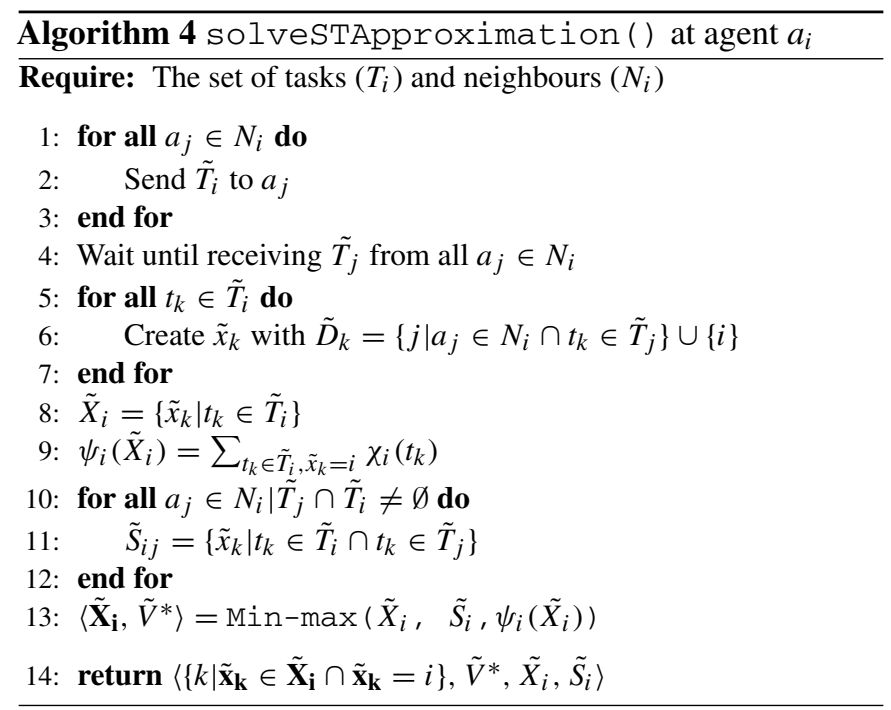

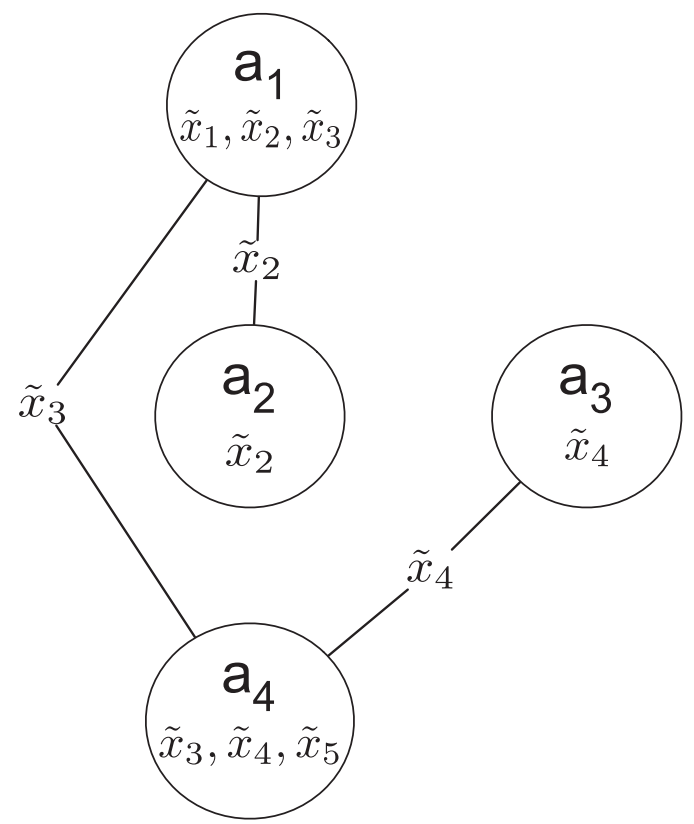

FIGURE 7. The JT formulation for the acyclic TDN resulting from the best-case heuristic shown in Fig. 4a. Large circles are cliques, with the elements of the cliques listed. Edges are labelled with common variables between cliques.

set of local variables to the set of variables representing the tasks in $\tilde{T}_{i}$ (line 8). Thirdly, the agent initializes its potential to encode its cost function incurred by tasks $\tilde{T}_{i}$ (line 9). Finally, the agent creates a separator for each neighbour with which it shares some task in the ST, containing the set of shared tasks (lines 10-12). Given its clique, potential and separators, each agent proceeds to run min-max as described in Section 2.2. As the outcome, each agent knows the optimal configuration of the ST for its local variables $\left(\tilde{\mathbf{X}}_{\mathbf{i}}^{*}\right)$ and its value $\left(\tilde{V}^{*}\right)$. The set of allocated tasks to the agent, $\left\{k \mid \tilde{\mathbf{x}}_{\mathbf{k}} \in \tilde{\mathbf{X}}_{\mathbf{i}} \cap \tilde{\mathbf{x}}_{\mathbf{k}}=i\right\}$, is computed from the optimal configuration (line 14).

As an example, Fig. 7 shows the JT computed by the agents during this step for the ST found by the best-case heuristic in Fig. 4 a. The domain of $\tilde{x}_{3}$ is restricted to 1,4 not including 2,3 
since these edges were pruned in the ST. Similarly, clique $\tilde{X}_{2}$ only contains $\tilde{x}_{2}$ since the possible allocation of $t_{3}$ to $a_{2}$ is not considered in the ST. After running min-max on this JT, it will find a solution where $x_{1}=1, x_{2}=2, x_{3}=1, x_{4}=3$ and $x_{5}=4$, and the makespan is $\max (10+50,20,70,60)=70$. Thus, the solution value found in this step, denoted by $\tilde{V}^{*}$, is 70 .

We can assess the complexity of this step from the complexity of running min-max over the JT encoding the acyclic TDN. As discussed at the end of Section 3.2, in acyclic TDNs the computation of the solution grows exponentially in the number of tasks an agent is connected to in the TDN. Since the TDNs that result from Step 1 have the maximum number of tasks that an agent can be connected to bounded by $\Delta$, min-max runs in polynomial time for fixed $\Delta$.

\subsection{Computing the per-instance approximation ratio}

The final step of ST-DTDA involves computing an approximation ratio, which can always be used to bound the quality of the solution found in step 2. Having an approximation ratio $\rho$ means that $\tilde{V}^{*} / V^{*} \leq \rho$ (e.g. the value of the solution found by ST-DTDA will not be more than a factor $\rho$ the value of the optimal solution).

Each agent estimates the approximation ratio $\rho$ of $\tilde{V}^{*}$ as follows:

$$
\rho=\frac{\tilde{V}^{*}}{\max \left(\tilde{V}^{*}-\varepsilon, V^{\mathrm{LB}}\right)},
$$

where $\varepsilon$ is an absolute bound on the error of $V^{*}\left(\tilde{V}^{*}-\varepsilon \leq V^{*}\right)$ and $V^{\mathrm{LB}}$ is a lower bound on $V^{*}\left(V^{\mathrm{LB}} \leq V^{*}\right)$. Note that $V^{*} \geq \max \left(\tilde{V}^{*}-\varepsilon, V^{\mathrm{LB}}\right)$ and then the approximation ratio from Equation (7) comes simply from rearranging this expression. We detail the computation of $\varepsilon$ and $V^{\mathrm{LB}}$ next.

Computing $\varepsilon$. Let $T^{M} \subseteq T$ be a set that contains tasks whose domains have been modified in the ST (i.e. at least one agenttask allocation has been omitted for each $t_{k} \in T^{M}$ ).

As proved in Appendix 2, we can define $\varepsilon$ as the value of the minimum makespan in the approximate problem composed only of tasks in $T^{M}$. Formally,

$$
\varepsilon=\min _{\tilde{X}} \max _{a_{i} \in A} \sum_{\substack{t_{k} \in T^{M} \\ \tilde{x}_{k}=i}} \chi_{i}\left(t_{k}\right) .
$$

To compute $\varepsilon$, ST-DTDA agents run min-max over the JT corresponding to the vertex-induced subgraph of the TDN induced by $T^{M}$ and the set of agents nodes.

Specifically, Algorithm 5 outlines the procedure that each ST-DTDA agent executes during this step. As input, each agent receives the set of variables and separators of the agent in the JT $\left(\tilde{X}_{i}, \tilde{S}_{i}\right)$, the value of the ST-DTDA solution $\left(\tilde{V}^{*}\right)$ and a lower bound $\left(V^{\mathrm{LB}}\right)$. Then, each agent proceeds to compute the JT corresponding to the vertex-induced subgraph of the TDN induced by $T^{M}$. First, each agent restricts the set of variables, and its clique, to those corresponding to tasks whose
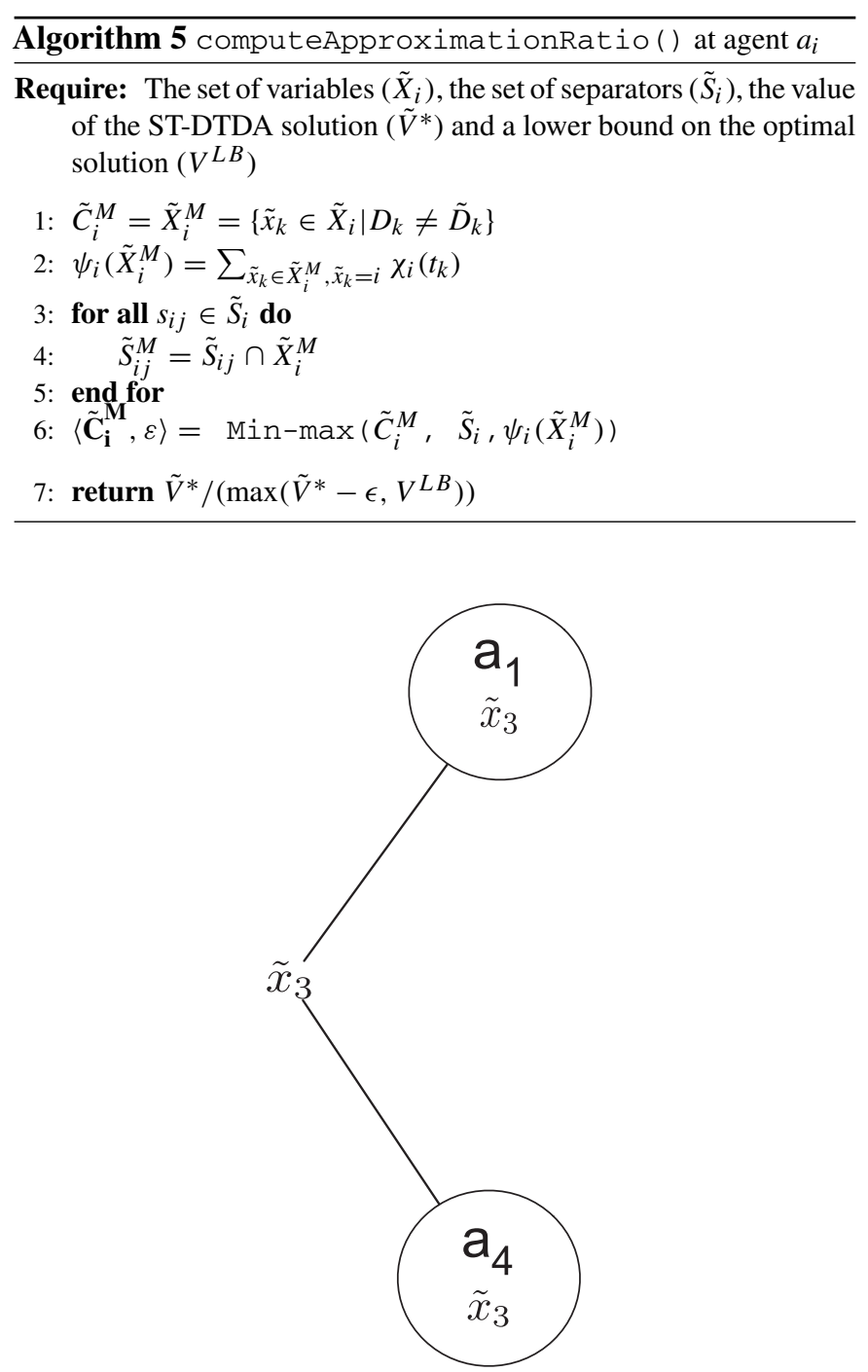

FIGURE 8. The JT whose solution gives a worst-case bound on the quality of the solution returned by ST-DTDA in the JT in Fig. 7. Edges are labelled with common variables between cliques.

domains have been modified in the ST (line 1). Secondly, each agent computes its potential as its cost function but considering only the task execution times of the modified tasks (line 2). Finally, each agent recomputes its separators (lines 3-5). Given its clique, potential and separators, each agent proceeds to run min-max. As the end of this procedure, each agent returns the approximation ratio computed as in Equation (7).

Figure 8 shows the JT the agents need to solve to obtain the worst-case bound for the solution produced by ST-DTDA over the JT specified in Fig. 7. The only edges removed in the ST found by the best-case in Fig. $4 \mathrm{a}$ are $\left(a_{2}, t_{3}\right)$ and $\left(a_{3}, t_{3}\right)$. Hence, in this case, $T^{M}=\left\{t_{3}\right\}$. To compute the bound, minmax is used to find the optimal value of $\tilde{x}_{3}$, which, in this case, is $a_{4}$ (e.g. $\left.\arg _{a_{1}, a_{4}} \min (50,30)=a_{4}\right)$. Note that, to compute the absolute error bound, ST-DTDA agents are only interested in 
the value of the best configuration, not in the best configuration itself. Thus, $\varepsilon=30$ in our example, which means that any solution found in step 3 will be at most 30 away from the optimal solution value.

Computing $V^{\mathrm{LB}}$. Now, there can be cases where $\varepsilon$ is equal to $\tilde{V}^{*}$. In particular, in cases where at least one edge per task needs to be pruned to create the ST. In such cases, there is no guarantee on the quality of the solution returned by agents running STDTDA because the value of the absolute error bound is the same than the value of the solution. To provide a guarantee in these cases, we combine this bound with a simple lower bound on the optimal solution, $V^{\mathrm{LB}}$. In particular, we define $V^{\mathrm{LB}}$ as the time taken for the fastest agent to perform the longest task. Formally,

$$
V^{\mathrm{LB}}=\max _{t_{k} \in T} \min _{a_{i} \in A} \chi_{i}\left(t_{k}\right) .
$$

Thus, for the TDN in Fig. $1, V^{\mathrm{LB}}$ is computed as

$$
\begin{aligned}
V^{\mathrm{LB}}= & \max \left(\min _{i \in\{1\}}\left(\chi_{i}\left(t_{1}\right)\right), \min _{i \in\{1,2\}}\left(\chi_{i}\left(t_{2}\right)\right), \min _{i \in\{1,2,3,4\}}\left(\chi_{i}\left(t_{3}\right)\right),\right. \\
& \left.\min _{i \in\{3,4\}}\left(\chi_{i}\left(t_{4}\right)\right), \min _{i \in\{4\}}\left(\chi_{i}\left(t_{5}\right)\right)\right) \\
= & \max (\min (10), \min (20,30), \min (50,60,80,30), \\
& \min (70,20), \min (60))=60 .
\end{aligned}
$$

Finally, given $\varepsilon=30, \tilde{V}^{*}=70$ and $V^{\mathrm{LB}}=60$, we compute the approximation ratio, according to Equation (7), as follows:

$$
\rho=\frac{70}{\max (70-30,60)}=\frac{70}{60}=1.16 .
$$

Thus, in this case, the best-case heuristic achieves an approximation ratio of 1.16 , despite the fact that the approximate solution is equal to the optimal.

We can assess the complexity of this step from the complexity of running min-max over the JT encoding the acyclic TDN restricted to tasks whose domains have been modified in the ST.

Thus, clearly the complexity of solving this JT is bounded above by the complexity of solving the JT for the whole acyclic TDN in Step 2.

Having completed our definition of ST-DTDA, we next proceed to evaluate it on a number of scenarios and compare it against a baseline algorithm in order to identify its strengths and weakness on different types of TDNs since its performance is clearly dependent on the graph structure (node degrees in particular) of the TDNs.

\section{EMPIRICAL EVALUATION}

In this section, we present an evaluation of the ST-DTDA algorithm (using our heuristics, namely best-case, worstcase and min-regret as given in Section 4.1). Moreover, we benchmark ST-DTDA against distributed stochastic algorithm
(DSA) ${ }^{18}$ a decentralized hill-climbing algorithm we propose as a baseline approach to solve Agent-Based $R \| C_{\max }$, and against IBM ILOG CPLEX,${ }^{19}$ a centralized mixed integer programming solver.

In what follows, first, we explain the details of our experimental setup in Section 5.1. Then, we describe and analyse our empirical results in Section 5.2.

\subsection{Experimental setup}

To determine the average-case performance of ST-DTDA and DSA, we evaluate them on random TDNs. The process of generating a TDN is divided in two steps. First, we generate the structure of the TDN, namely the set of agent/task nodes and the set of edges that stand for possible agent-task allocations. Secondly, we generate the task execution time for each edge between a task and an agent in the TDN.

As discussed in [7], the task-to-agent ratio and the density are the two key structural parameters that are most likely to impact performance. Hence, to control these parameters, the TDN is generated by specifying: the number of agents $(|A|)$, the number of tasks $(|T|)$ and the average number of tasks connected to an agent $\left(\left|T_{a}\right|\right)$. Thus, the first two parameters fix the taskto-agent ratio $(|T| /|A|)$ of the TDN whereas the latter fixes its density (or, equivalently, its sparsity). ${ }^{20}$ Then, a random tree can be generated with vertices composed of agents and tasks (this guarantees that the corresponding TDN is connected) where for each edge to be added, we randomly select one task and one agent not directly connected (i.e. there is no edge between that task and the agent) and add an edge between them. We then check that all tasks can potentially be executed by at least two agents. Otherwise, the instance is discarded. This check avoids the generation of trivial decision variables; a task with degree 1 generates a variable that can only take one value, that is, the only agent that can execute this task.

Once the structure of a TDN is generated, we proceed to generate the task execution time for each edge between a task and an agent. Now, as stated in [22], the solution quality of algorithms may depend on the method used to generate task execution times. To take this into account, we consider, for each instance, three different methods to generate the execution time of task $t_{k}$ at agent $a_{i}\left(\psi_{i}\left(t_{k}\right)\right)$ :

(i) Uncorrelated: $\psi_{i}\left(t_{k}\right)$ is drawn from a truncated normal distribution $N[100,10]$ bounded below ${ }^{21}$ by 10 ;

(ii) Agent correlated: times are correlated with the agents' computational delay. Thus, $\psi_{i}\left(t_{k}\right)$ is drawn from a

\footnotetext{
${ }^{18} \mathrm{~A}$ complete description of DSA algorithm is given in Appendix 1.

${ }^{19} \mathrm{https} / / / \mathrm{www} . \mathrm{ibm} . \mathrm{com} /$ software/commerce/optimization/cplexoptimizer/.

${ }^{20}$ From the number of agents and the average number of tasks connected to an agent, we can assess the number of edges included in the network $\left(|E|=|A| \cdot\left|T_{a}\right|\right)$.

${ }^{21}$ We use a bounded distribution to ensure that task execution times are never negative nor close to zero.
} 
truncated normal distribution $N\left[\alpha_{i}, 10\right]$ bounded below by 10 where $\alpha_{i}$, the agent's computational delay, is drawn from the uniform distribution $U[50,100]$;

(iii) Agent/task correlated: times are correlated with the agents' speed and the workload of the corresponding task. Thus, $\psi_{i}\left(t_{k}\right)$ is drawn from a truncated normal distribution $N\left[\beta_{k}+\alpha_{i}, 10\right]$ bounded below by 10 , where $\beta_{k}, \alpha_{i}$ stand, respectively, for the task's workload and the agent's delay. Both $\beta_{k}, \alpha_{i}$ are drawn from the uniform distribution $U[50,100]$.

Below we summarize the parameters used in our experiments:

(i) number of tasks: 100;

(ii) number of agents: 40, 60, 80;

(iii) number of tasks per agent: 5, 10, 15;

(iv) task execution times: uncorrelated, agent correlated and agent/task correlated;

(v) number of problems tested per combination: 100 .

For our benchmarks, we set the activation probability of DSA to 0.7 (a value that is reported to work well in [23] ${ }^{22}$ and the number of iterations to $10000\left(I_{\max }=10000\right),{ }^{23}$ while for CPLEX we use the version 12.4 and stop the computation after $900 \mathrm{~s}$. Finally, for ST-DTDA we set the maximum number of tasks per agent to $10(\Delta=10)$. All the experiments were performed on a $3.2 \mathrm{GHz}$ Intel Core i5 with $16 \mathrm{~GB}$ ram.

\subsection{Results}

Tables 1-3 list our results for each task execution time distribution. Each table contains an entry for each scenario, namely each combination of tasks $(|T|)$, agents $(|A|)$ and average number of tasks per agent $\left(\left|T_{a}\right|\right)$. The fourth column, headed by $m^{*} \backslash \check{m} /|T|$, reports the fraction of tasks in the optimal allocation, $m^{*}$, that differ from the greedy allocation, $\check{m}$, that assigns each task to the agent with minimal processing time. For each metric, we report the mean over 100 instances plus/minus the $95 \%$ confidence interval.

For CPLEX, we report the CPU time (in seconds) to capture the hardness of the problem when solved by a centralized algorithm. The number inside the parentheses is the number of problems that did not terminate within the time limit (CPU time averages are computed over the solved problems only). We observe that time taken by CPLEX to solve a problem can vary significantly across execution time distributions. Thus, CPLEX can solve most of the instances with uncorrelated times or times correlated with agents within a few seconds. In contrast, the same TDN structures with times correlated to agents and tasks tend to be computationally intensive. For instance, when

\footnotetext{
${ }^{22}$ We run DSA with different activation probabilities (i.e. 0.3 and 0.5 ) but results obtained were similar than the ones presented here for 0.7 .

${ }^{23}$ DSA always run this number of iterations, independently if its solution stabilize before.
}

$\left(|T|=100,|A|=60,\left|T_{a}\right|=15\right)$ more than one half of the instances (58) were not solved within the time limit (see Table 3).

For the rest of algorithms (ST-DTDA and DSA), they are extremely fast as expected and therefore we do not report computational times for them. In fact, all problems were solved within a few seconds and hence with much lower CPU requirements than CPLEX in many agent/task correlated instances. Instead, we focus our attention on the quality of their solutions. For each algorithm, we assess the quality of the solution it generates by dividing the optimal makespan found by CPLEX $\left(V^{*}\right)$ by the makespan of the solution found by the algorithm $\left(\tilde{V}^{*}\right){ }^{24}$ For ST-DTDA, Tables 1-3 also show the perinstance approximation ratio obtained in step 3 of ST-DTDA execution.

In general, we observe that the quality of the solutions found by ST-DTDA and DSA algorithms follow the same trend as the CPLEX solving times: instances with execution times agent-task correlated times lead to lower quality solutions than instances with uncorrelated or agent correlated times. For example, for uncorrelated times and the configuration $\left(|T|,|A|,\left|T_{a}\right|\right)=(100,40,15)$ (see Table 1$)$, the average solution quality of ST-DTDA with best-case is 0.57 . However, for the same configuration but with agent-task correlated times, the average solution quality decreases to 0.35 (see Table 3). A similar behaviour is observed for worst-case, min regret heuristics and DSA. To explain this result, we use the measure of the fraction of tasks that were not allocated in a greedy fashion in the optimal solution (i.e. the average fraction of tasks that were not assigned to the agent with minimal execution time), that is, $\left(m^{*} \backslash \breve{m}\right) /|T|$. We observe that the fraction of tasks not allocated in a greedy fashion is significantly higher in agent-task correlated times than in agent-correlated and uncorrelated times (e.g. compare the 0.55 of uncorrelated with the 0.79 of agenttask correlated for configuration $\left(|T|=100,|A|=80,\left|T_{a}\right|=\right.$ 15)). Hence, the first problems are more difficult than the latter (i.e. the higher the fraction of tasks allocations that differ from the greedy allocation, the lower the quality of the solutions of the algorithms).

On the other hand, although the number of tasks per agent affects the density of the TDN, this does not seem to have a significant impact on the solution quality of the algorithms for the parameters explored. Nevertheless, the quality of the solutions does vary significantly from one algorithm to the other as we analyse next.

First, we observe that ST-DTDA with best-case exhibits poor performance, especially for agent-task correlated times for which the average quality of the solutions ranges from 0.22 to 0.44 (see Table 3 ). This can be explained by the greedy behaviour of the best-case heuristic that assigns each task to the agents with the lowest processing times without taking into

\footnotetext{
${ }^{24}$ For problems in which CPLEX reaches the maximum time without finding the optimal solution, the best solution found up to this time limit is used.
} 
TABLE 1. Performance of ST-DTDA and DSA algorithms for uncorrelated execution times.

\begin{tabular}{|c|c|c|c|c|c|c|c|c|}
\hline \multicolumn{4}{|c|}{ Problem characteristics } & \multirow{2}{*}{$\begin{array}{l}\text { CPLEX } \\
\text { Time (s) }\end{array}$} & \multirow{2}{*}{$\begin{array}{l}\text { ST-DTDA best-case } \\
\frac{\text { Sol. Quality }}{\text { Approx. Ratio }}\end{array}$} & \multirow{2}{*}{$\begin{array}{l}\text { ST-DTDA worst-case } \\
\frac{\text { Sol. Quality }}{\text { Approx. Ratio }}\end{array}$} & \multirow{2}{*}{$\begin{array}{l}\text { ST-DTDA min-regret } \\
\frac{\text { Sol. Quality }}{\text { Approx. Ratio }}\end{array}$} & \multirow{2}{*}{$\frac{\text { DSA }}{\text { Sol. quality }}$} \\
\hline$|T|$ & $|A|$ & $\left|T_{a}\right|$ & $\frac{m^{*} \backslash \check{m}}{|T|}$ & & & & & \\
\hline \multirow[t]{9}{*}{100} & 40 & 5 & $0.40 \pm 0.01$ & $<1(0)$ & $\begin{array}{l}0.58 \pm 0.02 \\
4.45 \pm 0.13\end{array}$ & $\begin{array}{l}0.83 \pm 0.02 \\
3.05 \pm 0.06\end{array}$ & $\begin{array}{l}0.95 \pm 0.03 \\
2.66 \pm 0.02\end{array}$ & $0.72 \pm 0.04$ \\
\hline & & 10 & $0.44 \pm 0.01$ & $<1(0)$ & $\begin{array}{l}0.53 \pm 0.09 \\
4.85 \pm 0.16\end{array}$ & $\begin{array}{l}0.85 \pm 0.08 \\
3.00 \pm 0.06\end{array}$ & $\begin{array}{l}0.94 \pm 0.01 \\
2.63 \pm 0.02\end{array}$ & $0.76 \pm 0.04$ \\
\hline & & 15 & $0.46 \pm 0.01$ & $12 \pm 10(2)$ & $\begin{array}{l}0.57 \pm 0.02 \\
4.49 \pm 0.14\end{array}$ & $\begin{array}{l}0.89 \pm 0.01 \\
2.85 \pm 0.05\end{array}$ & $\begin{array}{l}0.93 \pm 0.00 \\
2.70 \pm 0.02\end{array}$ & $0.88 \pm 0.02$ \\
\hline & 60 & 5 & $0.44 \pm 0.01$ & $<1(0)$ & $\begin{array}{l}0.54 \pm 0.02 \\
3.29 \pm 0.12\end{array}$ & $\begin{array}{l}0.79 \pm 0.02 \\
2.24 \pm 0.06\end{array}$ & $\begin{array}{l}0.93 \pm 0.03 \\
1.85 \pm 0.01\end{array}$ & $0.89 \pm 0.01$ \\
\hline & & 10 & $0.46 \pm 0.04$ & $2 \pm 1(0)$ & $\begin{array}{l}0.50 \pm 0.09 \\
3.56 \pm 0.13\end{array}$ & $\begin{array}{l}0.81 \pm 0.11 \\
2.15 \pm 0.06\end{array}$ & $\begin{array}{l}0.94 \pm 0.01 \\
1.85 \pm 0.01\end{array}$ & $0.89 \pm 0.01$ \\
\hline & & 15 & $0.46 \pm 0.01$ & $3 \pm 0.46(0)$ & $\begin{array}{l}0.52 \pm 0.02 \\
3.47 \pm 0.12\end{array}$ & $\begin{array}{l}0.88 \pm 0.01 \\
2.00 \pm 0.04\end{array}$ & $\begin{array}{l}0.94 \pm 0.00 \\
1.86 \pm 0.01\end{array}$ & $0.90 \pm 0.01$ \\
\hline & 80 & 5 & $0.53 \pm 0.01$ & $<1(0)$ & $\begin{array}{l}0.59 \pm 0.02 \\
2.86 \pm 0.11\end{array}$ & $\begin{array}{l}0.89 \pm 0.01 \\
1.87 \pm 0.03\end{array}$ & $\begin{array}{l}0.93 \pm 0.03 \\
1.76 \pm 0.01\end{array}$ & $0.90 \pm 0.00$ \\
\hline & & 10 & $0.57 \pm 0.05$ & $14 \pm 35(0)$ & $\begin{array}{l}0.59 \pm 0.09 \\
2.92 \pm 0.10\end{array}$ & $\begin{array}{l}0.90 \pm 0.04 \\
1.87 \pm 0.03\end{array}$ & $\begin{array}{l}0.94 \pm 0.01 \\
1.77 \pm 0.01\end{array}$ & $0.90 \pm 0.00$ \\
\hline & & 15 & $0.55 \pm 0.01$ & $2 \pm 0.15(0)$ & $\begin{array}{l}0.60 \pm 0.02 \\
2.96 \pm 0.12\end{array}$ & $\begin{array}{l}0.93 \pm 0.01 \\
1.83 \pm 0.01\end{array}$ & $\begin{array}{l}0.94 \pm 0.00 \\
1.81 \pm 0.01\end{array}$ & $0.89 \pm 0.00$ \\
\hline
\end{tabular}

The number between parentheses in the CPLEX column is the number of instances not completed within $900 \mathrm{~s}$.

TABLE 2. Performance of ST-DTDA and DSA algorithms for agent correlated execution times.

\begin{tabular}{|c|c|c|c|c|c|c|c|c|}
\hline \multicolumn{4}{|c|}{ Problem characteristics } & \multirow{2}{*}{$\begin{array}{l}\text { CPLEX } \\
\text { Time (s) }\end{array}$} & \multirow{2}{*}{$\begin{array}{l}\text { ST-DTDA best-case } \\
\frac{\text { Sol. Quality }}{\text { Approx. Ratio }}\end{array}$} & \multirow{2}{*}{$\begin{array}{l}\text { ST-DTDA worst-case } \\
\frac{\text { Sol. Quality }}{\text { Approx. Ratio }}\end{array}$} & \multirow{2}{*}{$\begin{array}{l}\text { ST-DTDA min-regret } \\
\frac{\text { Sol. Quality }}{\text { Approx. Ratio }}\end{array}$} & \multirow{2}{*}{$\frac{\text { DSA }}{\text { Sol. quality }}$} \\
\hline$|T|$ & $|A|$ & $\left|T_{a}\right|$ & $\frac{m^{*} \backslash \check{m}}{|T|}$ & & & & & \\
\hline \multirow[t]{9}{*}{100} & 40 & 5 & $0.42 \pm 0.01$ & $<1(0)$ & $\begin{array}{l}0.58 \pm 0.02 \\
3.30 \pm 0.12\end{array}$ & $\begin{array}{l}0.80 \pm 0.01 \\
2.36 \pm 0.06\end{array}$ & $\begin{array}{l}0.93 \pm 0.01 \\
2.02 \pm 0.03\end{array}$ & $0.84 \pm 0.01$ \\
\hline & & 10 & $0.49 \pm 0.01$ & $<1(0)$ & $\begin{array}{l}0.54 \pm 0.01 \\
3.53 \pm 0.13\end{array}$ & $\begin{array}{l}0.81 \pm 0.01 \\
2.32 \pm 0.05\end{array}$ & $\begin{array}{l}0.92 \pm 0.00 \\
2.03 \pm 0.03\end{array}$ & $0.85 \pm 0.01$ \\
\hline & & 15 & $0.58 \pm 0.01$ & $<1(0)$ & $\begin{array}{l}0.52 \pm 0.01 \\
3.90 \pm 0.12\end{array}$ & $\begin{array}{l}0.85 \pm 0.01 \\
2.36 \pm 0.06\end{array}$ & $\begin{array}{l}0.89 \pm 0.00 \\
2.23 \pm 0.04\end{array}$ & $0.85 \pm 0.02$ \\
\hline & 60 & 5 & $0.49 \pm 0.01$ & $<1(0)$ & $\begin{array}{l}0.56 \pm 0.02 \\
2.58 \pm 0.01\end{array}$ & $\begin{array}{l}0.76 \pm 0.01 \\
1.85 \pm 0.04\end{array}$ & $\begin{array}{l}0.89 \pm 0.01 \\
1.57 \pm 0.03\end{array}$ & $0.80 \pm 0.01$ \\
\hline & & 10 & $0.58 \pm 0.01$ & $<1(0)$ & $\begin{array}{l}0.50 \pm 0.01 \\
2.88 \pm 0.11\end{array}$ & $\begin{array}{l}0.78 \pm 0.02 \\
1.85 \pm 0.05\end{array}$ & $\begin{array}{l}0.90 \pm 0.00 \\
1.58 \pm 0.03\end{array}$ & $0.82 \pm 0.01$ \\
\hline & & 15 & $0.64 \pm 0.01$ & $<1(0)$ & $\begin{array}{l}0.48 \pm 0.01 \\
3.50 \pm 0.12\end{array}$ & $\begin{array}{l}0.83 \pm 0.01 \\
1.99 \pm 0.04\end{array}$ & $\begin{array}{l}0.87 \pm 0.01 \\
1.88 \pm 0.04\end{array}$ & $0.85 \pm 0.01$ \\
\hline & 80 & 5 & $0.58 \pm 0.01$ & $<1(0)$ & $\begin{array}{l}0.52 \pm 0.02 \\
2.18 \pm 0.10\end{array}$ & $\begin{array}{l}0.76 \pm 0.02 \\
1.50 \pm 0.05\end{array}$ & $\begin{array}{l}0.90 \pm 0.01 \\
1.23 \pm 0.02\end{array}$ & $0.79 \pm 0.01$ \\
\hline & & 10 & $0.65 \pm 0.01$ & $<1(0)$ & $\begin{array}{l}0.46 \pm 0.01 \\
2.75 \pm 0.10\end{array}$ & $\begin{array}{l}0.80 \pm 0.02 \\
1.56 \pm 0.05\end{array}$ & $\begin{array}{l}0.90 \pm 0.00 \\
1.39 \pm 0.03\end{array}$ & $0.82 \pm 0.01$ \\
\hline & & 15 & $0.69 \pm 0.01$ & $<1(0)$ & $\begin{array}{l}0.46 \pm 0.01 \\
3.21 \pm 0.14\end{array}$ & $\begin{array}{l}0.87 \pm 0.01 \\
1.66 \pm 0.04\end{array}$ & $\begin{array}{l}0.87 \pm 0.01 \\
1.64 \pm 0.03\end{array}$ & $0.83 \pm 0.01$ \\
\hline
\end{tabular}

The number between parentheses in the CPLEX column is the number of instances not completed within $900 \mathrm{~s}$. 
TABLE 3. Performance of ST-DTDA and DSA algorithms for agent/task correlated execution times.

\begin{tabular}{|c|c|c|c|c|c|c|c|c|}
\hline \multicolumn{4}{|c|}{ Problem characteristics } & \multirow{2}{*}{$\begin{array}{l}\text { CPLEX } \\
\text { Time }(\mathrm{s})\end{array}$} & \multirow{2}{*}{$\begin{array}{l}\text { ST-DTDA best-case } \\
\frac{\text { Sol. Quality }}{\text { Approx. Ratio }}\end{array}$} & \multirow{2}{*}{$\begin{array}{l}\text { ST-DTDA worst-case } \\
\frac{\text { Sol. Quality }}{\text { Approx. Ratio }}\end{array}$} & \multirow{2}{*}{$\begin{array}{l}\text { ST-DTDA min-regret } \\
\frac{\text { Sol. Quality }}{\text { Approx. Ratio }}\end{array}$} & \multirow{2}{*}{$\frac{\text { DSA }}{\text { Sol. quality }}$} \\
\hline$|T|$ & $|A|$ & $\left|T_{a}\right|$ & $\frac{m^{*} \backslash \check{m}}{|T|}$ & & & & & \\
\hline \multirow[t]{9}{*}{100} & 40 & 5 & $0.55 \pm 0.01$ & $11 \pm 12(1)$ & $\begin{array}{l}0.44 \pm 0.01 \\
4.61 \pm 0.12\end{array}$ & $\begin{array}{l}0.74 \pm 0.01 \\
2.74 \pm 0.05\end{array}$ & $\begin{array}{l}0.88 \pm 0.01 \\
2.29 \pm 0.02\end{array}$ & $0.67 \pm 0.03$ \\
\hline & & 10 & $0.60 \pm 0.01$ & $152 \pm 39(41)$ & $\begin{array}{l}0.39 \pm 0.01 \\
5.04 \pm 0.12\end{array}$ & $\begin{array}{l}0.74 \pm 0.01 \\
2.65 \pm 0.04\end{array}$ & $\begin{array}{l}0.87 \pm 0.00 \\
2.29 \pm 0.03\end{array}$ & $0.65 \pm 0.03$ \\
\hline & & 15 & $0.68 \pm 0.01$ & $205 \pm 31(20)$ & $\begin{array}{l}0.35 \pm 0.01 \\
5.77 \pm 0.12\end{array}$ & $\begin{array}{l}0.75 \pm 0.01 \\
2.69 \pm 0.05\end{array}$ & $\begin{array}{l}0.85 \pm 0.00 \\
2.37 \pm 0.03\end{array}$ & $0.73 \pm 0.03$ \\
\hline & 60 & 5 & $0.62 \pm 0.01$ & $2 \pm 0.60(0)$ & $\begin{array}{l}0.38 \pm 0.10 \\
4.04 \pm 0.14\end{array}$ & $\begin{array}{l}0.73 \pm 0.01 \\
2.05 \pm 0.04\end{array}$ & $\begin{array}{l}0.86 \pm 0.01 \\
1.74 \pm 0.02\end{array}$ & $0.81 \pm 0.01$ \\
\hline & & 10 & $0.70 \pm 0.01$ & $72 \pm 29(8)$ & $\begin{array}{l}0.31 \pm 0.01 \\
4.91 \pm 0.14\end{array}$ & $\begin{array}{l}0.73 \pm 0.01 \\
2.05 \pm 0.04\end{array}$ & $\begin{array}{l}0.87 \pm 0.01 \\
1.80 \pm 0.02\end{array}$ & $0.80 \pm 0.01$ \\
\hline & & 15 & $0.78 \pm 0.01$ & $232 \pm 54(58)$ & $\begin{array}{l}0.27 \pm 0.01 \\
5.68 \pm 0.14\end{array}$ & $\begin{array}{l}0.74 \pm 0.01 \\
2.07 \pm 0.03\end{array}$ & $\begin{array}{l}0.87 \pm 0.01 \\
1.77 \pm 0.02\end{array}$ & $0.79 \pm 0.01$ \\
\hline & 80 & 5 & $0.66 \pm 0.01$ & $9 \pm 14(1)$ & $\begin{array}{l}0.32 \pm 0.01 \\
3.80 \pm 0.14\end{array}$ & $\begin{array}{l}0.69 \pm 0.01 \\
1.76 \pm 0.04\end{array}$ & $\begin{array}{l}0.81 \pm 0.01 \\
1.48 \pm 0.02\end{array}$ & $0.76 \pm 0.01$ \\
\hline & & 10 & $0.74 \pm 0.01$ & $7 \pm 4(2)$ & $\begin{array}{l}0.25 \pm 0.01 \\
4.87 \pm 0.15\end{array}$ & $\begin{array}{l}0.69 \pm 0.01 \\
1.77 \pm 0.03\end{array}$ & $\begin{array}{l}0.81 \pm 0.01 \\
1.53 \pm 0.02\end{array}$ & $0.76 \pm 0.01$ \\
\hline & & 15 & $0.79 \pm 0.01$ & $12 \pm 6(2)$ & $\begin{array}{l}0.22 \pm 0.01 \\
5.65 \pm 0.15\end{array}$ & $\begin{array}{l}0.71 \pm 0.01 \\
1.75 \pm 0.03\end{array}$ & $\begin{array}{l}0.78 \pm 0.01 \\
1.59 \pm 0.02\end{array}$ & $0.76 \pm 0.01$ \\
\hline
\end{tabular}

The number between parentheses in the CPLEX column is the number of instances not completed within $900 \mathrm{~s}$.

account the fact that these agents may already be overloaded. In contrast, as expected, ST-DTDA with worst-case and minregret achieves significantly higher quality (around 0.69-0.75 and $0.78-0.88$, respectively, with agent-task correlated times, see Table 3). This is explained by the fact that the worstcase and min-regret heuristics consider the current agents' workload as defined by the best allocation of the existing tasks connected to the agents. Thus, these heuristics can better minimize the possibility of overloading some agents and therefore creating bottlenecks. Secondly, we find that the minregret and worst-case heuristics have very similar performances but the quality of the solutions of min-regret is always higher (around 5-15\% higher). This is because the min-regret heuristic prioritized the tasks with higher regret. Finally, DSA returns competitive solutions, sometimes better than ST-DTDA with the worst-case heuristic on average. Nevertheless, DSA always achieves worse average solutions than ST-DTDA with min-regret.

Finally, regarding the per-instance approximation ratio we observe that in general the approximation ratio returned by STDTDA is not very accurate (i.e. there is a significant gap between the real approximation ratio of the solution found and the one reported by ST-DTDA). For instance, observe in Table 2, that the average approximation ratio returned by ST-DTDA with $\mathrm{min}$ regret for configuration $\left(|T|=100,|A|=40,\left|T_{a}\right|=5\right)$ is 2.02 whilst its real average approximation ratio is $\frac{1}{0.93}=1.07$.
We also observe that the accuracy of the bound gets worse as we reduce the number of agents in the problem. For instance, the average approximation ratio returned by ST-DTDA with min-regret for the same configuration $(|T|=100,|A|=80$, $\left|T_{a}\right|=5$ ) but with 80 agents is 1.23 (see Table 2), much closer to it real average approximation ratio, $\frac{1}{0.90}=1.11$.

Overall, the best results in each scenario are achieved by STDTDA with min-regret. On all configurations, ST-DTDA is thus able to achieve high quality solutions ( $>0.78$ of the optimal) and outperform the baseline algorithm.

\section{CONCLUSIONS AND FUTURE WORK}

In this paper, we have presented ST-DTDA, the first decentralized algorithm for the problem of scheduling a set of tasks on unrelated parallel machines (i.e. $R \| C_{\max }$ ). In more detail, ST-DTDA uses the min-max message-passing algorithm to compute an approximate solution for the AgentBased $R \| C_{\max }$ problem in a completely decentralized manner. In so doing, ST-DTDA agents execute three steps. First, they use a heuristic approach that deletes possible agent-task allocations in order to form an acyclic version of the original problem. In particular, we define, and evaluate, three different heuristics for weighting the importance of links between agents and tasks they can do. Secondly, agents run the min-max 
message-passing algorithm to compute the optimal solution to this relaxed $R \| C_{\max }$ problem. Finally, they compute a perinstance approximation ratio for its solution.

Our empirical evaluation shows that ST-DTDA with the minregret heuristic is able to produce high-quality solutions $(>78 \%$ of the optimal) on a range of network structures and distributions of task processing times. In particular, we show that the minregret heuristics that recompute weights during the formation of the relaxed $R \| C_{\text {max }}$ problem and prioritizes the allocations with respect to the regret leads to typically high quality solutions. Finally, we benchmark ST-DTDA against a distributed hillclimbing approach and show that it outperforms it in all tested configurations.

We believe ST-DTDA is a significant first step in the space of decentralized solutions to the unrelated parallel machines problem with machine eligibility restrictions. Yet, the bounding of complexity of ST-DTDA by means of $\Delta$ only makes STDTDA truly scalable in terms of solution quality in domains where the interactions between the tasks and machines are sparse (i.e. each agent can execute a small number of tasks and each task can be executed by a small number of agents). Otherwise, in very dense problems, the restriction imposed by $\Delta$ is likely to lead to many tasks single-connected in the ST and hence, with its allocation fixed already in the first step. Hence, future work will look into extensions that make it scalable and provide more accurate bounds in domains where agenttask interactions are not as sparse as those evaluated in this paper. Finally, in this paper we focused on scheduling problems where the set of tasks are independent. Hence, in future, we intend to extend our approach to deal with problems with task precedence constraints (i.e. where one or more tasks may have to be completed before another task is allowed to start its processing) as in [24].

\section{FUNDING}

This work was supported by the iDEaS (www.ideasproject.info) and ORCHID (www.orchid.ac.uk) projects at the University of Southampton.

\section{REFERENCES}

[1] Graham, R.L., Lawler, E.L., Lenstra, J.K. and Rinnoy Kan, A.H.G. (1979) Optimization and approximation in deterministic sequencing and scheduling: a survey. Ann. Discrete Math., 5, 287-326.

[2] Pinedo, M.L. (2008) Scheduling: Theory, Algorithms, and Systems (3rd edn). Springer.

[3] Horowitz, E. and Sahni, S. (1976) Exact and approximate algorithms for scheduling nonidentical processors. J. ACM, 23, 317-327.

[4] Shchepin, E.V. and Vakhania, N. (2005) An optimal rounding gives a better approximation for scheduling unrelated machines. Oper. Res. Lett., 33, 127-133.
[5] Lenstra, J.K., Shmoys, D.B. and Tardos, E. (1990) Approximation algorithms for scheduling unrelated parallel machines. Math. Program., 46, 259-271.

[6] Wooldridge, M. and Jennings, N.R. (1995) Intelligent agents: theory and practice. Knowl. Eng. Rev., 10, 115-152.

[7] Wotzlaw, A. (2006) Scheduling unrelated parallel machines: algorithms, complexity, and performance. $\mathrm{PhD}$ Thesis, Universität Paderborn.

[8] Jensen, F.V. and Nielsen, T.D. (2007) Bayesian Networks and Decision Graphs (2nd edn). Springer.

[9] Aji, S.M. and McEliece, R.J. (2000) The generalized distributive law. IEEE Trans. Inf. Theory, 46, 325-343.

[10] Leung, J.Y.-T. and Li, C.-L. (2008) Scheduling with processing set restrictions: a survey. Int. J. Prod. Econ., 116, 251-262.

[11] Farinelli, A., Vinyals, M., Rogers, A. and Jennings, N.R. (2013) Distributed Search and Constraint Handling. In Weiss, G. (ed.), Multiagent Systems: A Modern Approach to Distributed Artificial Intelligence. MIT Press.

[12] Fitzpatrick, S. and Meertens, L. (2002) Experiments on Dense Graphs with a Stochastic, Peer-to-Peer Colorer. Probabilistic Approaches in Search, Workshop at 18th National Conf. on Artificial Intelligence (AAAI), Alberta, Canada, pp. 24-28. AAAI Press.

[13] Farinelli, A., Rogers, A. and Jennings, N.R. (2009) Bounded Approximate Decentralised Coordination using the Maxsum Algorithm. At the 21st Int. Joint Conf. on Artificial Intelligence (IJCAI), Pasadena, California, USA, July, pp. 46-59.

[14] Vinyals, M., Rodríguez-Aguilar, J.A. and Cerquides, J. (2011) Constructing a unifying theory of dynamic programming DCOP algorithms via the generalized distributive law. J. Auton. Agents Multi-Agent Syst., 22, 439-464.

[15] Pouly, M. and Kohlas, J. (2011) Generic Inference: A Unifying Theory for Automated Reasoning (1st edn). Wiley.

[16] Wainwright, M.J. and Jordan, M.I. (2008) Graphical models, exponential families, and variational inference. Found. Trends Mach. Learn., 1, 1-305.

[17] Collin, Z. and Dolev, S. (1994) Self-stabilizing depth-first search. Inf. Process. Lett., 49, 297-301.

[18] Paskin, M.A., Guestrin, C. and McFadden, J. (2005) A Robust Architecture for Distributed Inference in Sensor Networks. Proc. 4th Int. Conf. Information Processing in Sensor Networks (IPSN), UCLA, Los Angeles, California, USA, April, pp. 55-62. IEEE.

[19] Vinyals, M., Rodriguez-Aguilar, J.A. and Cerquides, J. (2009) Generalizing DPOP: Action-GDL, A New Complete Algorithm for DCOPs. Proc. 8th Int. Joint Conf. Autonomous Agents and Multiagent Systems (AAMAS), Budapest, Hungary, May, pp. 1239-1240. IFAAMAS.

[20] Rogers, A., Farinelli, A., Stranders, R. and Jennings, N.R. (2011) Bounded approximate decentralised coordination via the maxsum algorithm. Artif. Intell., 175, 730-759.

[21] Kruskal, J.B. (1956) On the shortest spanning subtree of a graph and the traveling salesman problem. Proc. Amer. Math. Soc., 7, $48-50$. 
[22] Vredeveld, T. and Hurkens, C.A.J. (2002) Experimental comparison of approximation algorithms for scheduling unrelated parallel machines. INFORMS J. Comput., 14, 175-189.

[23] Zhang, W., Wang, G., Xing, Z. and Wittenburg, L. (2005) Distributed stochastic search and distributed breakout: properties, comparison and applications to constraint optimization problems in sensor networks. Artif. Intell., 161, 55-87.

[24] Satish, N., Ravindran, K. and Keutzer, K. (2007) A Decomposition-Based Constraint Optimization Approach for Statically Scheduling Task Graphs with Communication Delays to Multiprocessors. Design, Automation and Test in Europe Conf. Exposition (DATE), Nice, France, April 16-20, pp. 57-62. ACM.

\section{APPENDIX 1. A BASELINE APPROACH TO DECENTRALIZED $R \| C_{\mathrm{MAX}}$}

As stated in Section 2, there are no decentralized algorithms for $R \| C_{\max }$ in the literature. Given this, we describe a baseline benchmarking approach to decentralized $R \| C_{\max }$ that results from applying a standard distributed hill-climbing algorithm to this problem. More concretely, this algorithm is inspired by the DSA, formulated in [12] for distributed constraint optimization problems. The key idea behind DSA is that each variable iteratively selects (with some probability), the value that minimizes its cost given the values chosen by other variables in the last iteration.

In more detail, Algorithm A.1 describes our implementation of DSA for $R \| C_{\max }$. Since decisions in $R \| C_{\max }$ correspond to which agent we assign each task to, we initially assign a group of tasks to each agent $a_{i} \in A$ for which they are responsible for computing an assignment. We denote this as $P_{i}$. The decision as to partition the tasks does not influence the final task allocation. Hence, we use a simple rule where each shared task goes to the agent with the lowest ID. Agents also agree on the maximum number of iterations, $I_{\max }$, that they will run the algorithm for. Finally, DSA uses an activation threshold, $p_{A}$, to reduce the likelihood that multiple changes occur simultaneously with outdated information, a phenomenon that results from the decentralization. Thus, Algorithm A.1 receives $P_{i}, I_{\max }$ and $p_{A}$ as inputs.

Following Algorithm A.1, each agent starts by running the procedure initialize (Algorithm A.1, lines 1-7). First, the agent's assigned task set, $m\left(a_{i}\right)$, is initialized to the set of tasks for which the agent should find an allocation (line 2). Then, for each task, the agent initializes its iteration counter, denoted as $I\left(t_{k}\right)$, to 0 (line 4) and sends a request message to each of the agents which can be assigned that task asking for their marginal costs (line 5).

When an agent $a_{i}$ receives a REQUEST $\left(t_{k}\right)$ message, it runs the procedure given in lines 8-11 to compute its marginal cost for task $t_{k}$ (given the assignment of all other tasks). The marginal cost of an agent $a_{i}$ for a task $t_{k}$, namely $\Lambda_{i k}$, is computed as the sum of the time it takes $a_{i}$ to compute $t_{k}$ plus the total time it would take $a_{i}$ to compute all tasks assigned to it in the

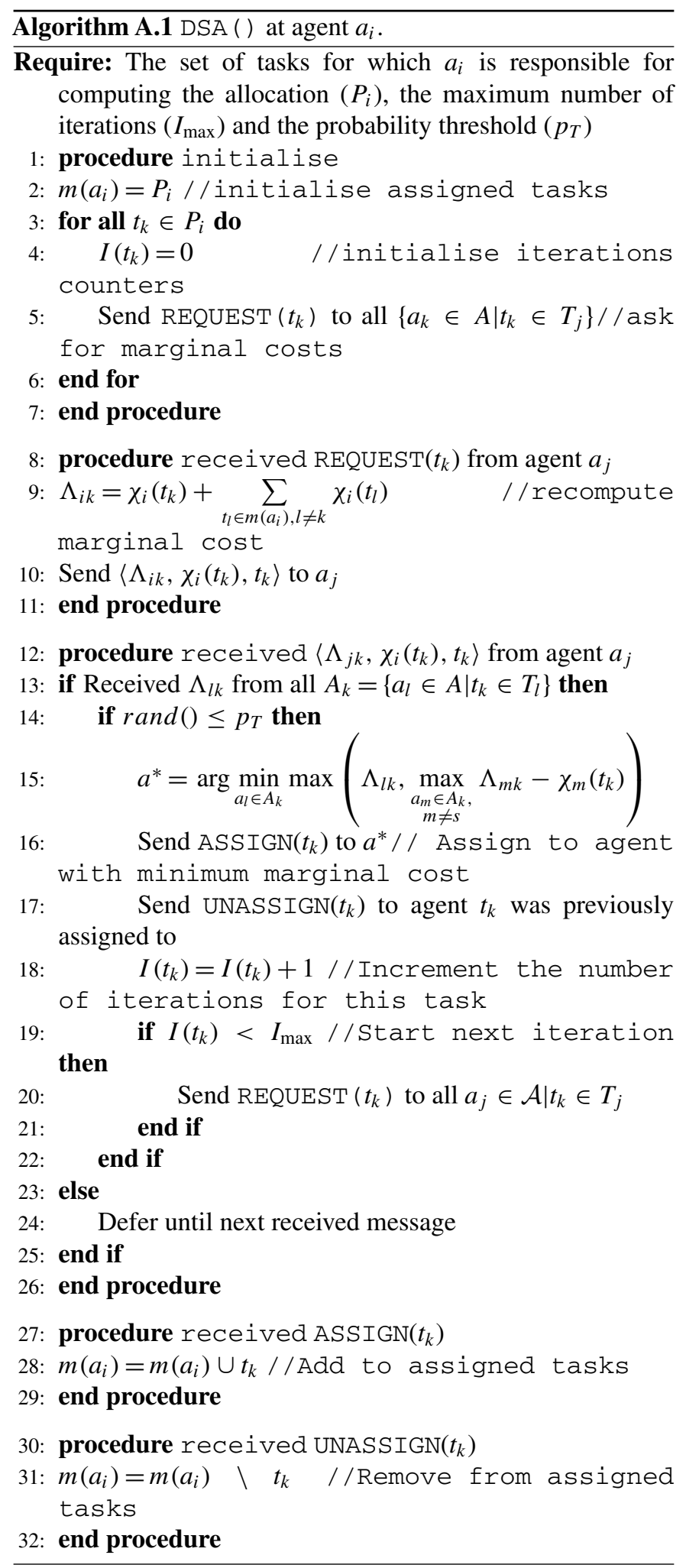

current assignment (line 9). This value, along with the time it takes $a_{i}$ to compute $t_{k}$, is then sent back to the requesting agent (line 10). 


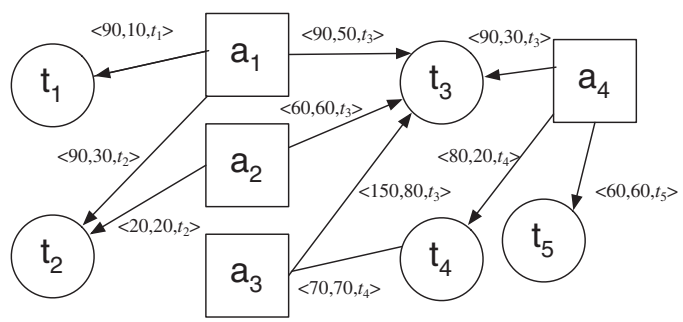

FIGURE A1. Agents respond to REQUEST messages during the first iteration of DSA algorithm over the TDN depicted in Fig. 1.

Once an agent has received the marginal costs from all agents to which a task $t_{k}$ can be assigned (line 13), it proceeds to consider changing the current assignment of this task with a probability $p_{A}$ (line 14). Thus, if the generated random number (given by the rand() function) is lower than $p_{A}$, the agent proceeds to compute the best assignment by finding the agent that minimizes the cost of the assignment. The cost of assigning an agent $a_{l}$ to task $t_{k}$ is computed as the maximum between this agent's marginal cost, $\Lambda_{l k}$, and the marginal cost of the rest of the agents $\left\{a_{m}, t_{k} \in T_{m}, m \neq l\right\}$, when excluding this assignment, $\Lambda_{m k}-\chi_{m}\left(t_{k}\right)$ (line 15). Agent $a_{i}$ sends an ASSIGN $\left(t_{k}\right)$ message to the agent with the minimum cost of assignment (line 16) and an UNASSIGN $\left(t_{k}\right)$ message to the agent to which $t_{k}$ is currently assigned (line 17). Then, the iteration counter is incremented (line 18), and, if the algorithm has not reached the maximum number of iterations, the process starts over again (lines 19-21). Once all iterations have been completed for all tasks $t_{k} \in T$, each agent has a set of tasks assigned by the algorithm.

Figure A1 shows a single iteration of the first step of DSA over the TDN in Fig. 1. In this example, agent $a_{1}$ is initially assigned tasks $t_{1}, t_{2}$ and $t_{3}$ for which it is in charge of computing the allocation. Similarly, $t_{4}$ is assigned to $a_{3}$ and $t_{5}$ to $a_{4}$. Agents begin by sending a REQUEST message, effectively from each task to each agent that can compute it (following the links agent-task in Fig. 1) and on receipt of a REQUEST $\left(t_{k}\right)$ from another agent, each agent computes its $\Lambda_{i k}$ value as detailed in Algorithm A.1, and sends that back, along with the task processing cost, to the sender for $t_{k}$ (this is denoted by the text on the arrows in Fig. A1). Observe that, for example, the marginal cost of assigning $t_{2}$ to $a_{1}$ is 90 , even when the execution time of $t_{2}$ is 30 , because in the initial assignment agent $a_{1}$ executes $t_{1}$ and $t_{3}$.

\section{APPENDIX 2. PROOFS OF BOUNDED APPROXIMATION}

In this appendix, we give the proofs for the absolute bound $\varepsilon$. Namely, we prove that

$$
\tilde{V}^{*}-V^{*} \leq\left[\varepsilon=\min _{\tilde{X}} \max _{a_{i} \in A} \sum_{\substack{t_{k} \in T^{M} \\ \tilde{x}_{k}=i}} \chi_{i}\left(t_{k}\right)\right] .
$$

To prove Equation (A.1), consider the subset of assignments of variables in $\tilde{X}$ in which the set of tasks in $T^{M}$ (e.g. tasks whose domain have been modified with respect to the original problem) are assigned to the same agent as they are assigned to in the optimal solution. That is, any assignment $\tilde{X}^{\alpha}$ such that $x_{i}^{\alpha}=x_{i}^{*}$ if $t_{i} \notin T^{M}$. Note that since $\tilde{X}^{\alpha}$ is an assignment of variables $\tilde{X}$, the value of any $\tilde{X}^{\alpha}, \tilde{V}^{\alpha}$, will always be higher (worse) than the optimal value of the ST approximate problem, $\tilde{V}^{*}$. Then, it follows that $\forall \tilde{X}^{\alpha}$ :

$$
\begin{aligned}
\tilde{V}^{*}-V^{*} \leq & \tilde{V}^{\alpha}-V^{*} \\
= & \max _{a_{i} \in A}\left(\sum_{\substack{t_{k} \in T^{M} \\
x_{k}^{*}=i}} \chi_{i}\left(t_{k}\right)+\sum_{\substack{t_{k^{*} \notin T^{M}} \\
\tilde{x}_{k}^{\alpha}=i}} \chi_{i}\left(t_{k}\right)\right) \\
& -\max _{a_{i} \in A}\left(\sum_{\substack{t_{k} \in T \\
x_{k}^{*}=i}} \chi_{i}\left(t_{k}\right)\right) \\
\leq & \max _{a_{i} \in A}\left(\sum_{\substack{t_{k} \notin T^{M} \\
\tilde{x}_{k}^{T}=i}} \chi_{i}\left(t_{k}\right)-\sum_{\substack{t_{k} \notin T^{M} \\
x_{k}^{*}=i}} \chi_{i}\left(t_{k}\right)\right) \\
\leq & \max _{a_{i} \in A}\left(\sum_{\substack{t_{k} \notin T^{M} \\
\tilde{x}_{k}^{M}=i}} \chi_{i}\left(t_{k}\right)\right.
\end{aligned}
$$

Thus, the value of the makespan of any assignment of tasks in $T^{M}$ to agents in the ST problem bounds the distance of $\tilde{V}^{*}$ to the optimal $V^{*}$. This also holds for the particular assignment of tasks $T^{M}$ that minimizes the makespan. Thus, Equation (A.1) holds. 